\title{
Prospects for Higgs Boson Searches at the LHC
}
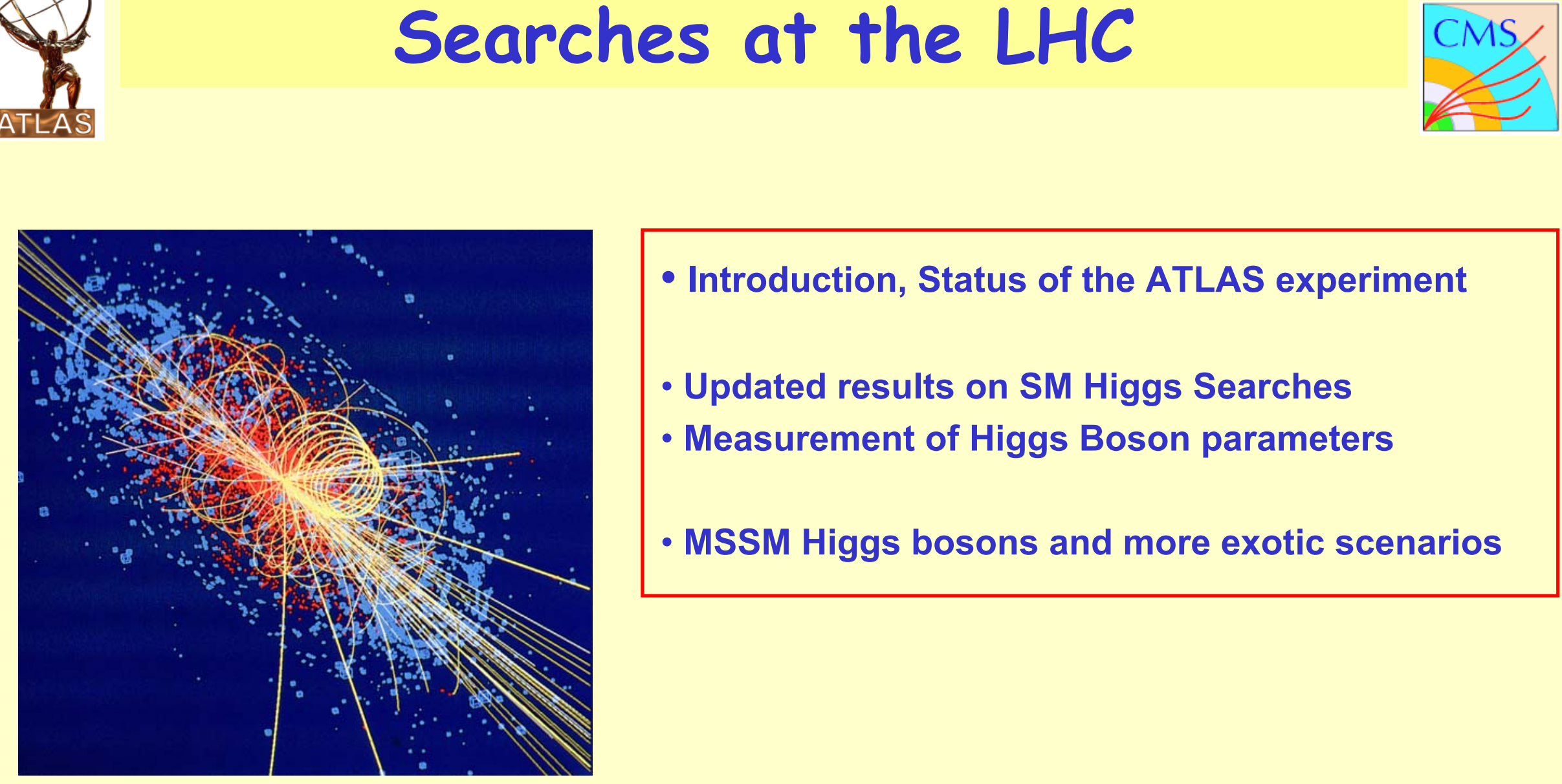

- Introduction, Status of the ATLAS experiment

- Updated results on SM Higgs Searches

- Measurement of Higgs Boson parameters

- MSSM Higgs bosons and more exotic scenarios

Karl Jakobs

Physikalisches Institut

Universität Freiburg / Germany

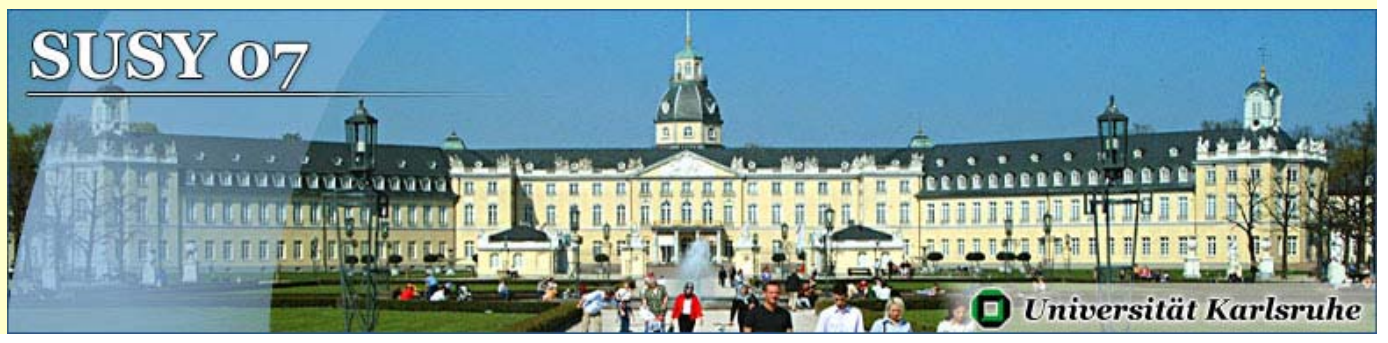




\section{The Higgs Boson}

- "Revealing the physical mechanism that is responsible for the breaking of electroweak symmetry is one of the key problems in particle physics"

- "A new collider, such as the LHC must have the potential to detect this particle, should it exist."

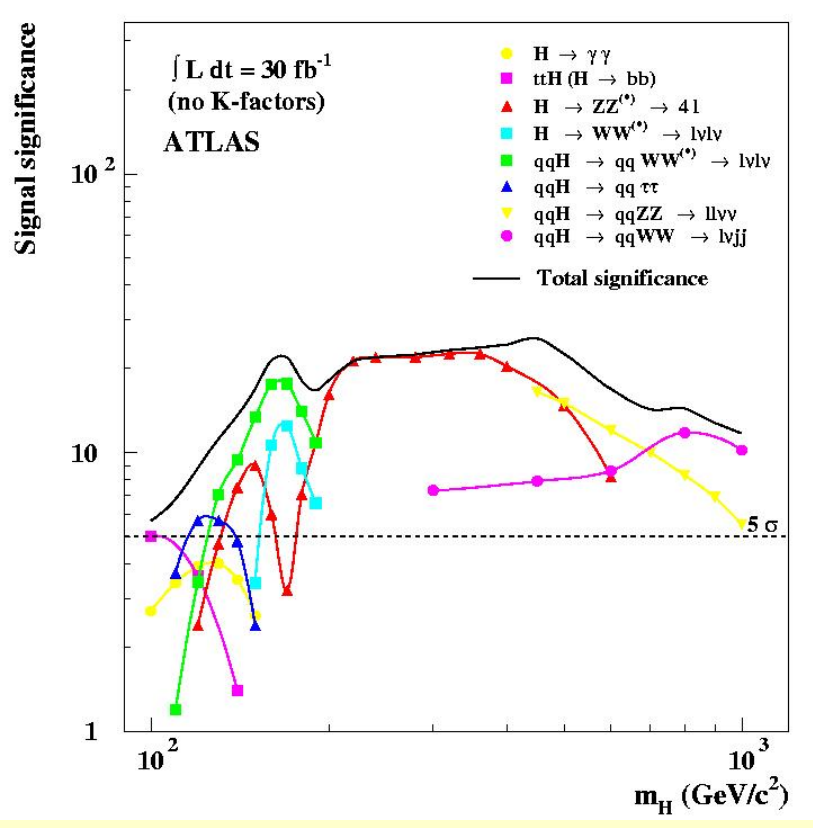

O. Buchmüller et al., arXiv:0707.3447

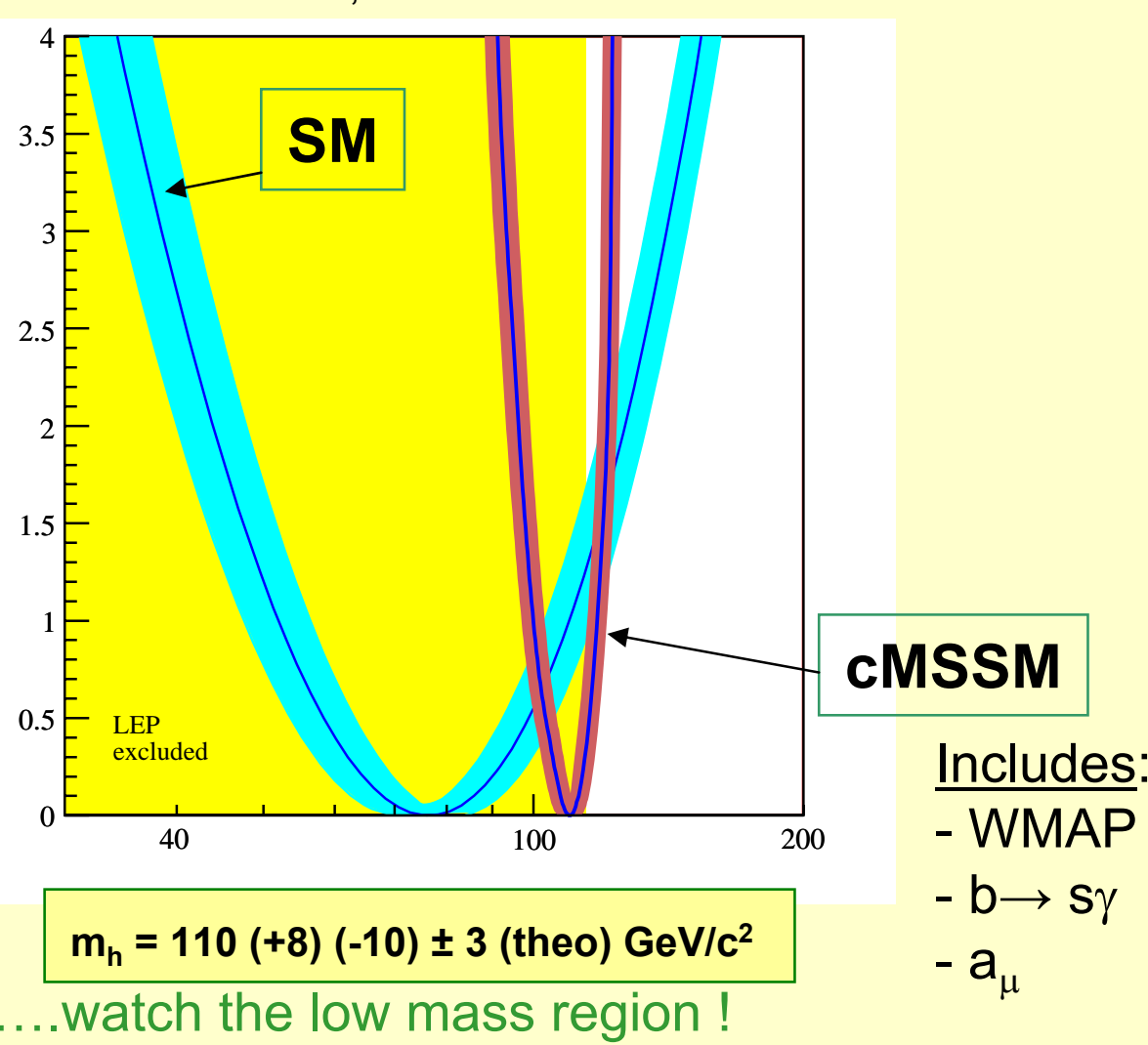

...expected to be achieved (ATLAS \& CMS studies) 


\section{Status of the ATLAS Installation}

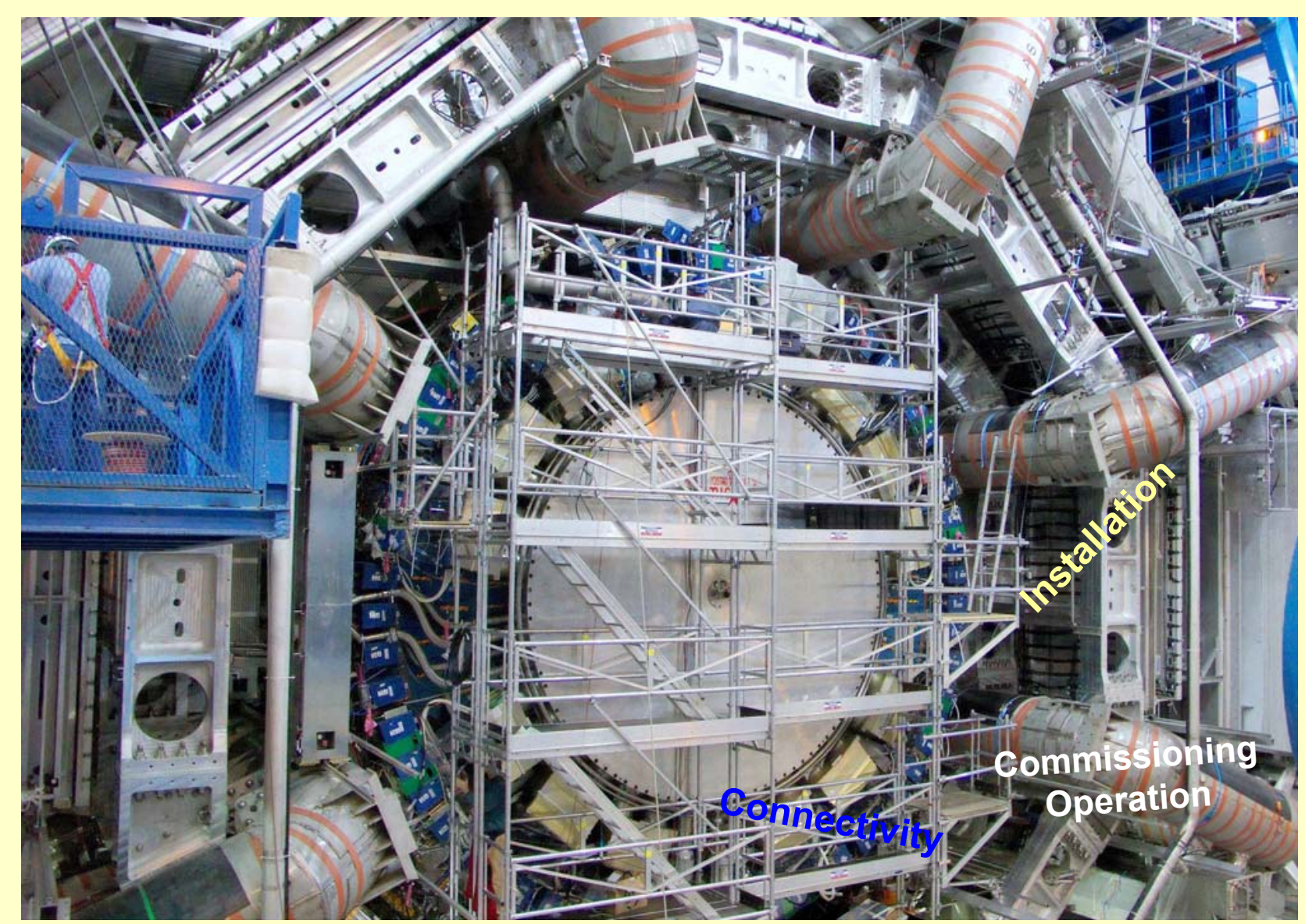

Calorimeters in place, since Sept. 2006

- Installation and Commissioning of the detector in full swing

- ATLAS will be ready for first pp collisions in Summer 2008 
Installation of one of the ATLAS Endcap Tracking Detectors (completed on 29. May 2007)

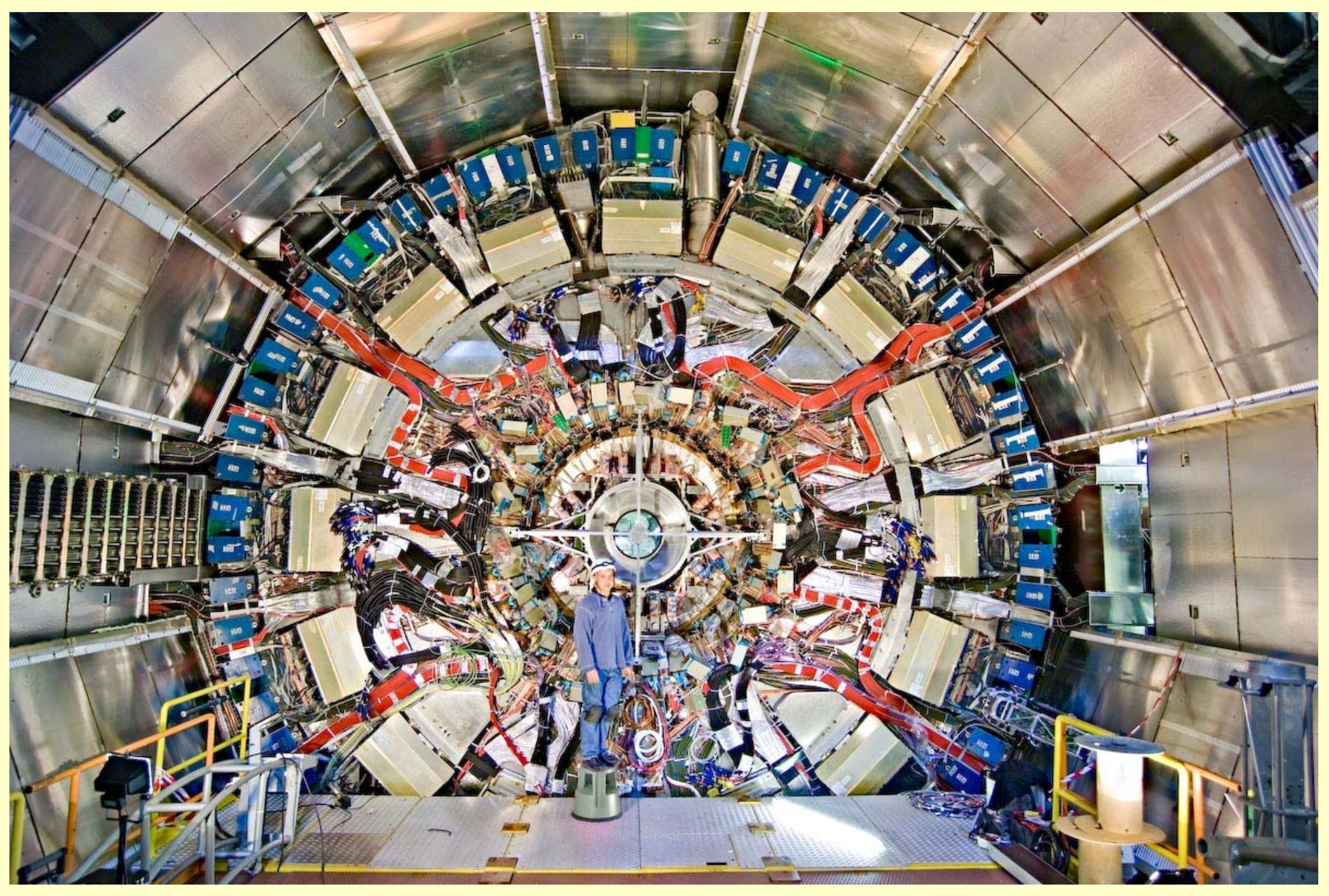




\section{Installation of Inner Detector Services}

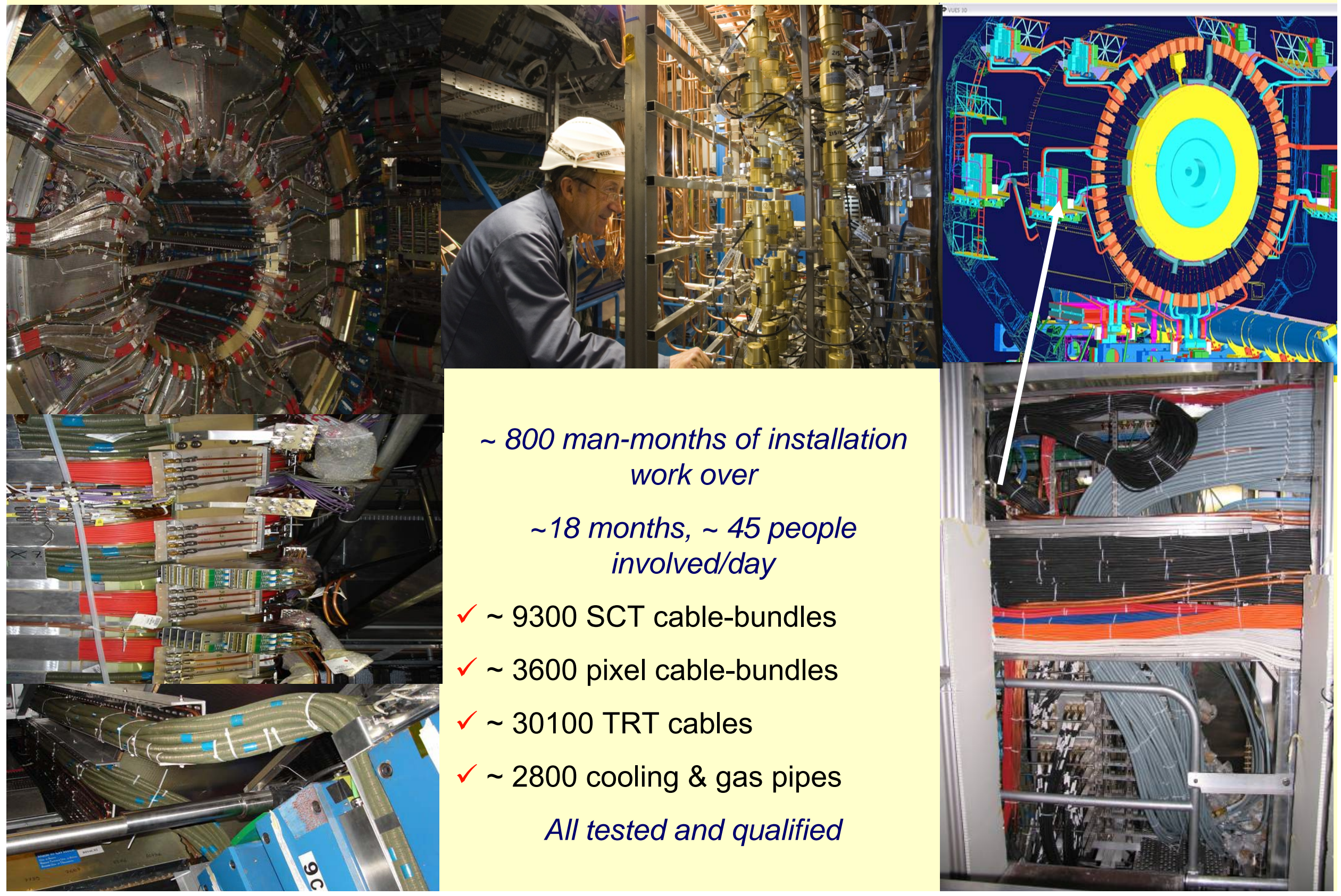




\section{Muon detectors and endcap toroid magnets}

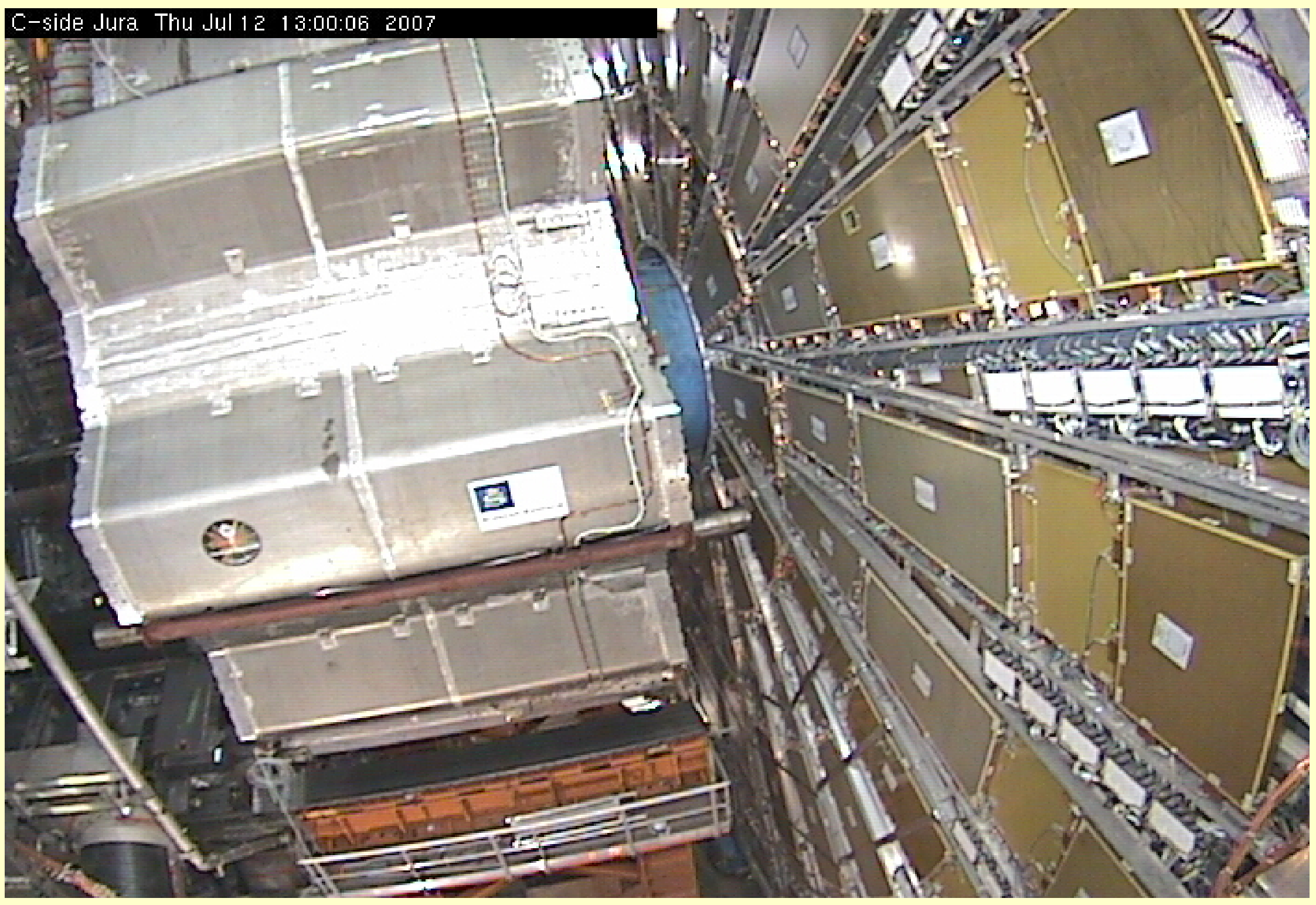

Installation of the second (last) endcap toroid: 12. July 07 


\section{What is new on LHC Higgs studies?}

- Many studies have meanwhile been performed using detailed GEANT simulations of the detectors

- Physics Performance Technical Design Report

from the CMS collaboration

- ATLAS CSC (Computing System Challenge) notes in

preparation, to be released towards the end of 2007

- New (N)NLO Monte Carlos (also for backgrounds)

- MCFM Monte Carlo, J. Campbell and K. Ellis, http://mcfm.fnal.gov

- MC@NLO Monte Carlo, S.Frixione and B. Webber, wwwweb.phy.cam.ar.uk/theory/

- T. Figy, C. Oleari and D. Zeppenfeld, Phys. Rev. D68, 073005 (2003)

- E.L.Berger and J. Campbell, Phys. Rev. D70, 073011 (2004)

- C. Anastasiou, K. Melnikov and F. Petriello, hep-ph/0409088 and hep-ph/0501130

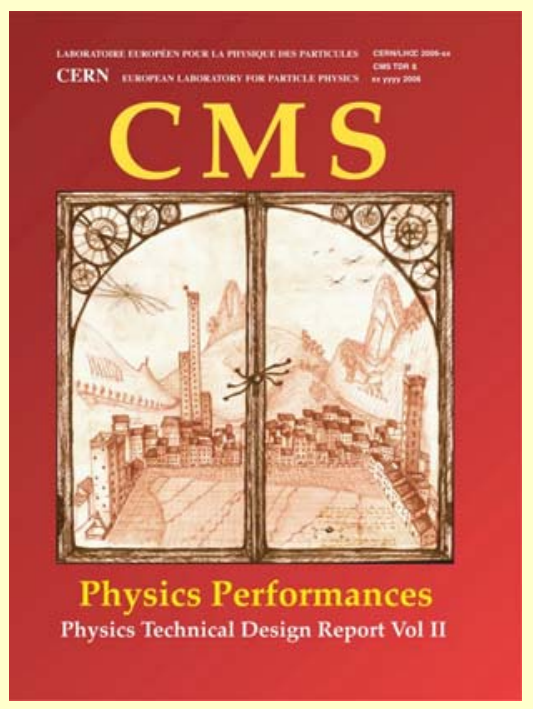

$-\ldots$.

- New approaches to match parton showers and matrix elements

- ALPGEN Monte Carlo + MLM matching, M. Mangano et al.

- SHERPA Monte Carlo, F. Krauss et al.

$-\ldots$

Tevatron data are extremely valuable for validation, work has started

- More detailed, better understood reconstruction methods

(partially based on test beam results,...)

- Further studies of new Higgs boson scenarios

(Various MSSM benchmark scenarios, CP-violating scenarios, Invisible Higgs boson decays,.....) 


\section{Standard Model}

\section{Higgs Boson Searches}

NLO cross sections, M.Spira et al.
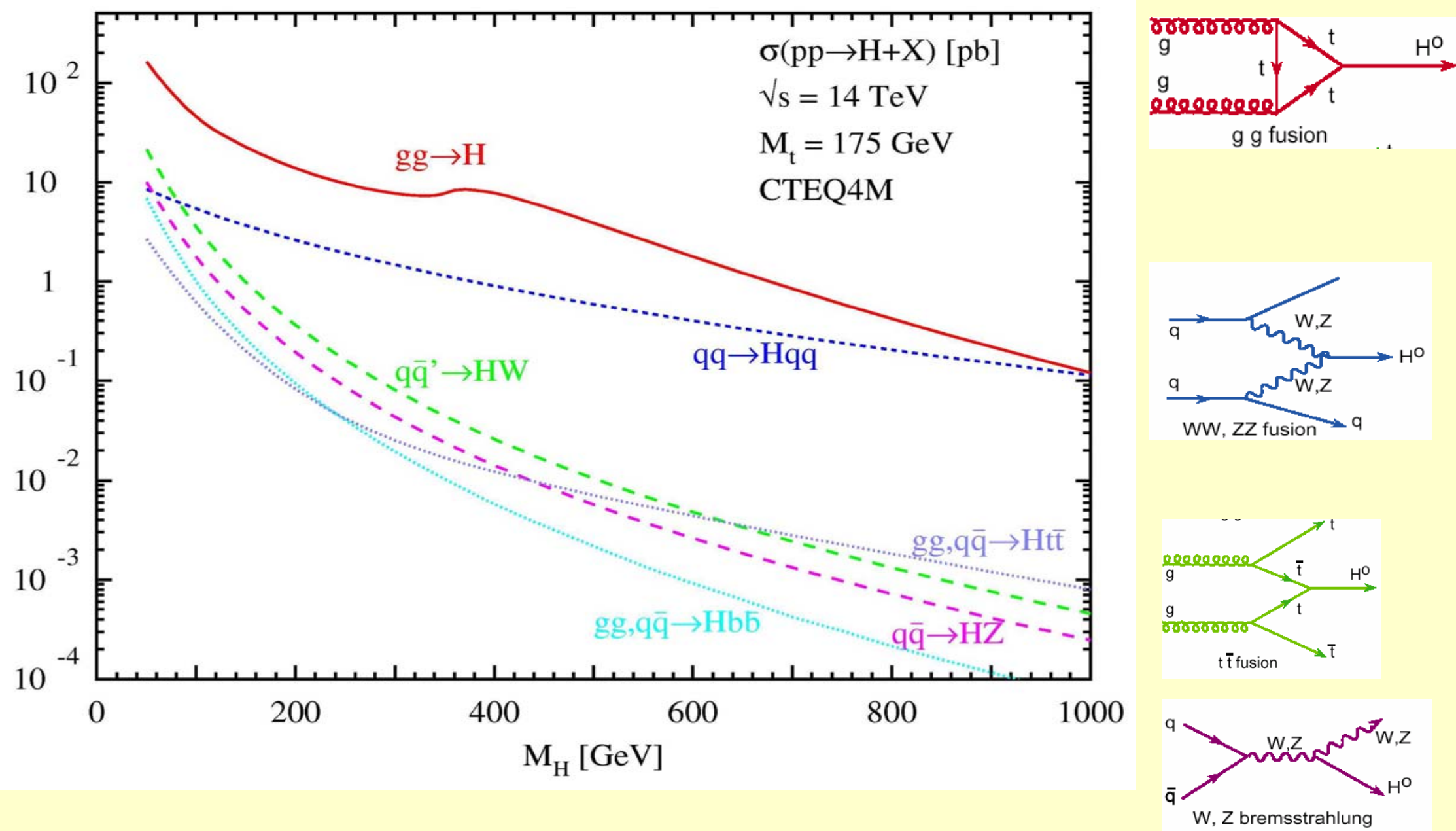


\section{$\underline{\mathrm{H} \rightarrow \mathrm{ZZ}^{*} \rightarrow \mathrm{el} \mathrm{el}}$}

- Main backgrounds: ZZ (irreducible), tt, Zbb (reducible)

- Main experimental tools for background suppression: - lepton isolation in the tracker and in the calorimeter - impact parameter

\section{Updated CMS study:}

- ZZ background: NLO K factor used

- background from side bands (gg->ZZ is added as $20 \%$ of the LO qq->ZZ)

Signal and background at $5 \sigma$ discovery
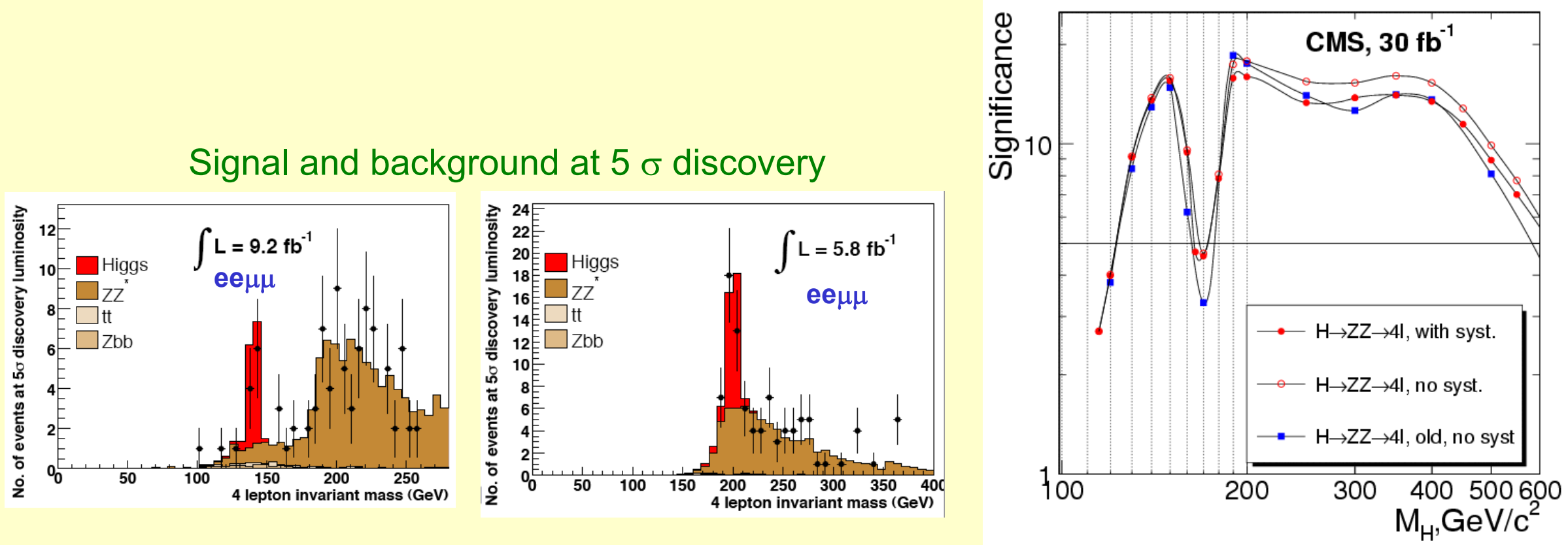


\section{Main backgrounds:}

\section{$\underline{\mathbf{H} \rightarrow \gamma \gamma}$}

$\gamma \gamma$ irreducible background

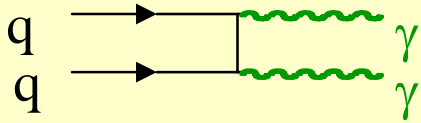

$\gamma$-jet and jet-jet (reducible)
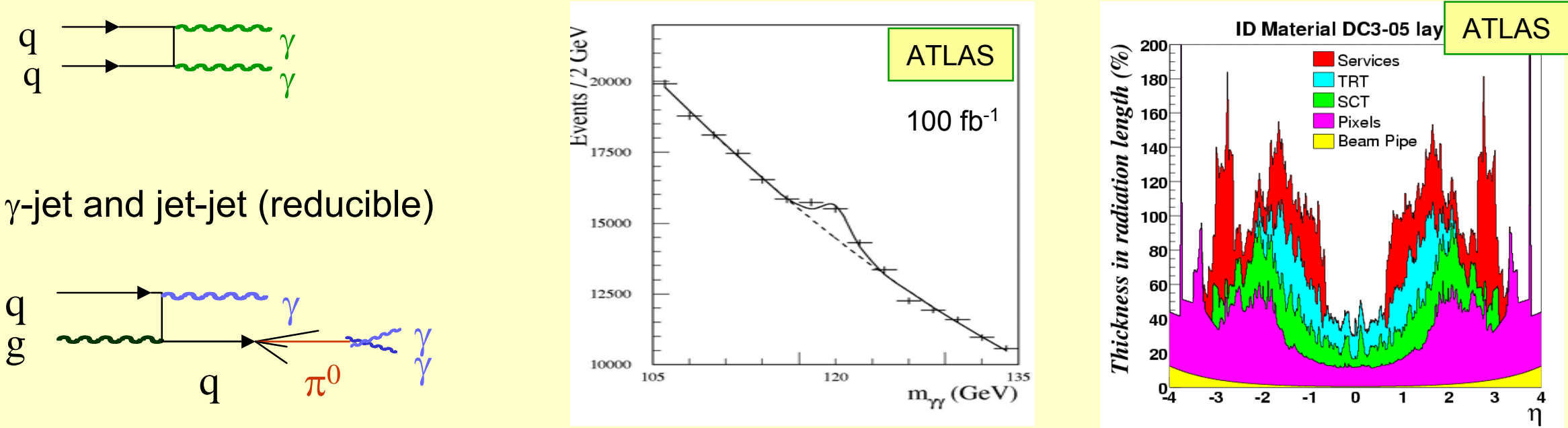

$\sigma_{\gamma j+j j} \sim 10^{6} \sigma_{\gamma \gamma} \quad$ with large uncertainties

$\rightarrow$ need $\mathrm{R}_{\mathrm{j}}>10^{3}$ for $\varepsilon_{\gamma} \approx 80 \%$ to get

$$
\sigma_{\gamma j+j \mathrm{j}} \ll \sigma_{\gamma \gamma}
$$

- Main exp. tools for background suppression:

- photon identification

$-\gamma /$ jet separation (calorimeter + tracker)

- note: also converted photons need to be reconstructed (large material in LHC silicon trackers)

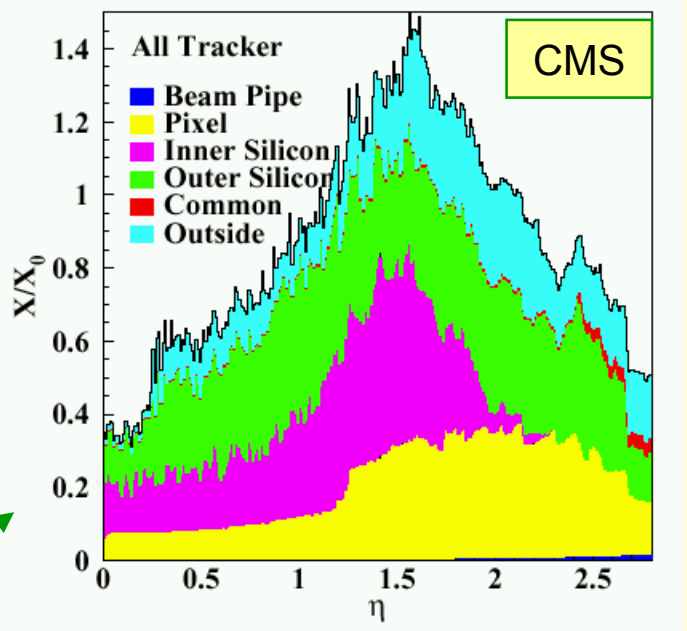

CMS: fraction of converted $\gamma_{\mathrm{s}}$ Barrel region: $\quad 42.0 \%$

Endcap region: $\quad 59.5 \%$ 


\section{CMS Study: TDR (updated)}

New elements of the analysis:

- more contributions to the $\gamma \gamma$ background
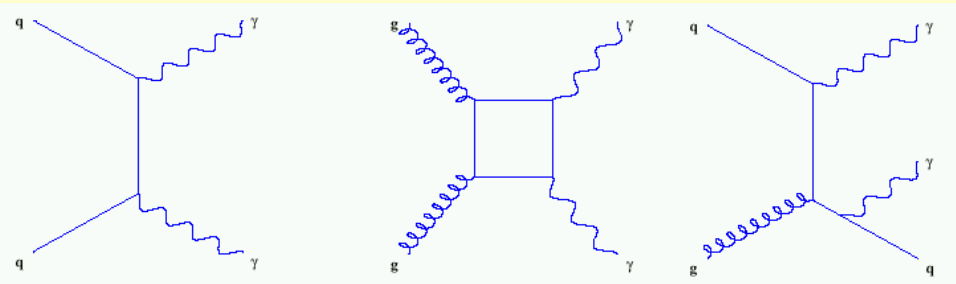

- NLO calculations available (Binoth et al., DIPHOX, RESBOS)

- Realistic detector material

- More realistic K factors (for signal and background)

- Split signal sample acc. to resolution functions

- Improvements possible by using more exclusive $\gamma \gamma+$ jet topologies

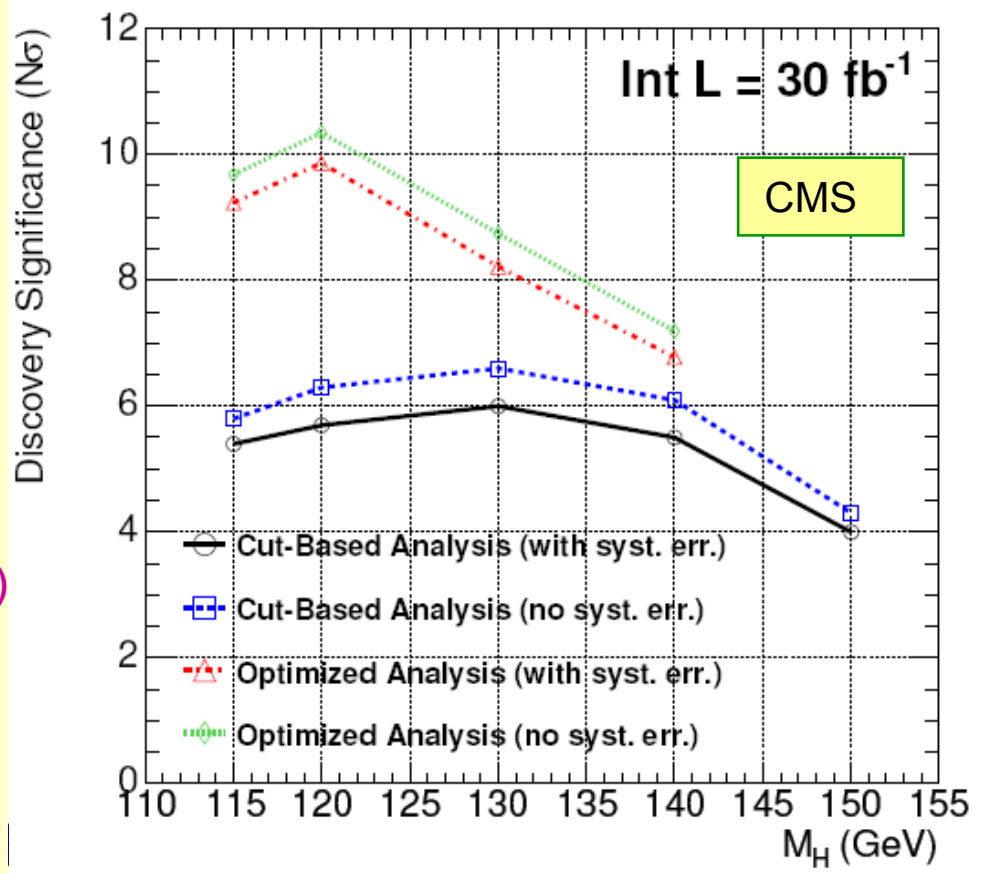

Signal significance for $m_{H}=130 \mathrm{GeV} / c^{2}$ and $30 \mathrm{fb}^{-1}$

\begin{tabular}{|l|l|l|}
\hline ATLAS & LO (TDR, 1999) & $3.9 \sigma$ \\
& NLO (update, cut based) & $6.3 \sigma$ \\
& NLO (likelihood methods) & $8.7 \sigma$ \\
\hline \multirow{2}{*}{ CMS } & NLO (cut based, TDR-2006) & $6.0 \sigma$ \\
& NLO (neural net optimization, TDR-2006) & $8.2 \sigma$ \\
\hline
\end{tabular}

Comparable results for ATLAS and CMS 


\section{$\underline{H} \rightarrow W W \rightarrow \ell_{V} \ell_{V}$}

- Large $\mathrm{H} \rightarrow$ WW $B R$ for $m_{H} \sim 160 \mathrm{GeV} / \mathrm{c}^{2}$

- Neutrinos $\rightarrow$ no mass peak, $\rightarrow$ use transverse mass

- Large backgrounds: WW, Wt, tt

- Two main discriminants:

(i) Lepton angular correlation

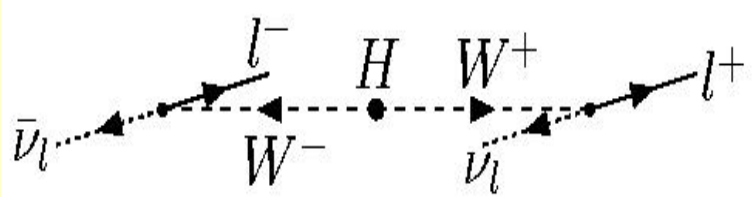

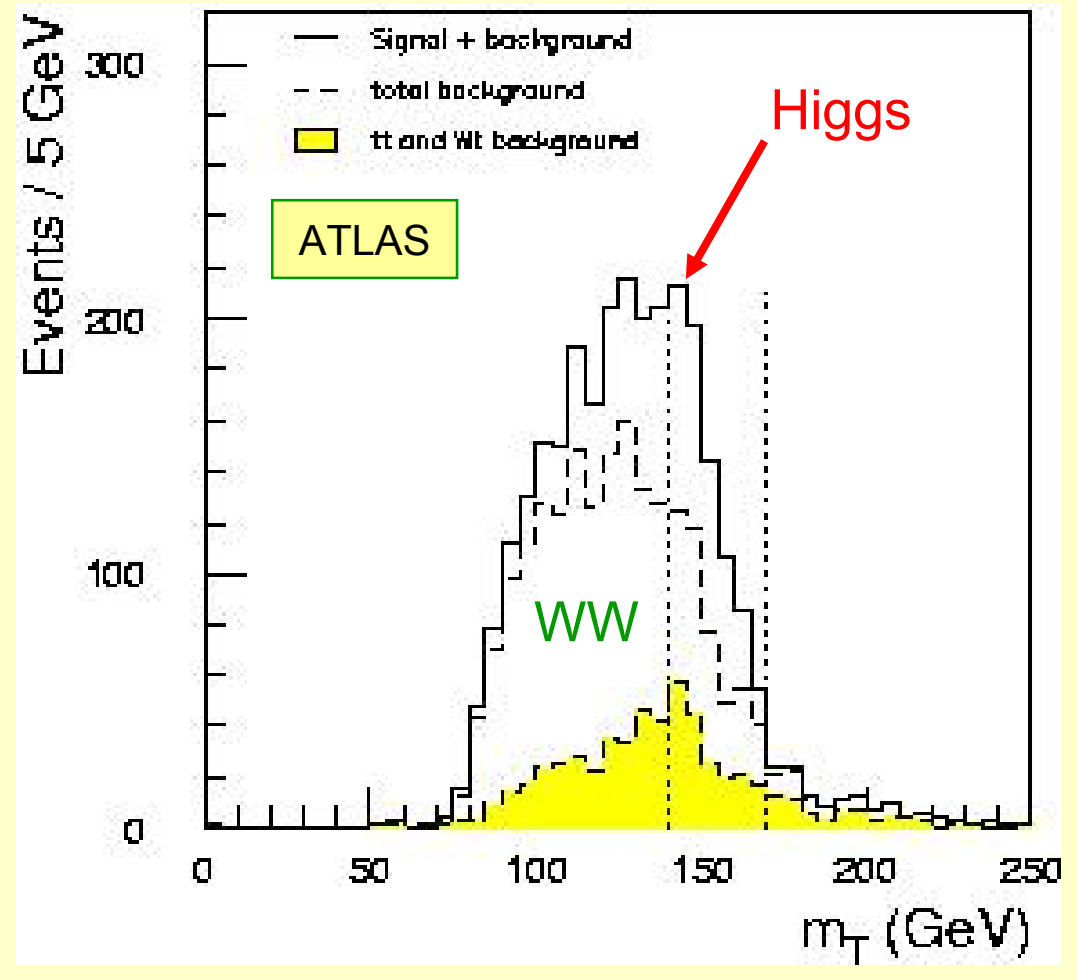

(ii) Jet veto: no jet activity in central detector region

\section{Difficulties:}

(i) need precise knowledge of the backgrounds Strategy: use control region(s) in data, extrapolation in signal region

(ii) jet veto efficiencies need to be understood for signal and background events $\rightarrow$ reliable Monte Carlo generators 


\section{Discovery reach in $\mathrm{H} \rightarrow W W \rightarrow \ell_{v} \ell_{v}$}

New developments:

- $g \mathrm{~g} \rightarrow$ WW box contribution found to be important

Small cross section (5\% of WW backgr.) before cuts, but $\Delta \phi$ shape similar to signal (30\% contribution after cuts)

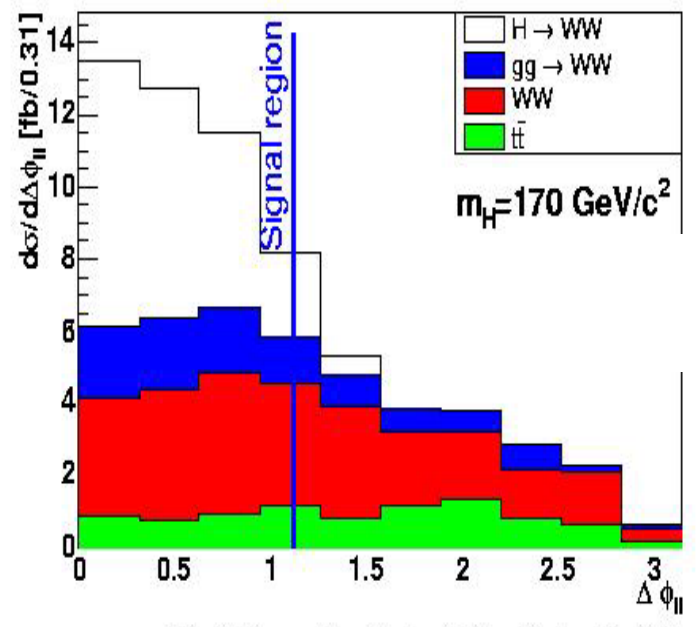

M. Dührssen et al., hep-ph/0504006

- Include both tt and single $\mathrm{t}$ background at NLO (Les Houches 2005)

\section{CMS Phys. TDR 2006}

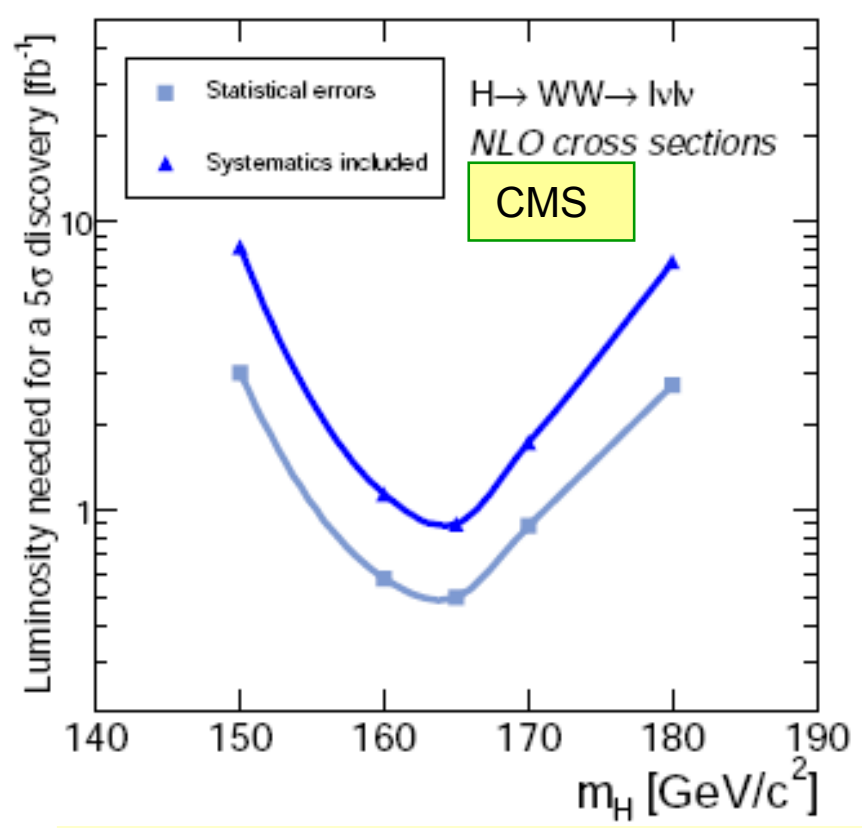

LHC:

luminosity needed for a $5 \sigma$ discovery

Estimated background uncertainties:

- tt from data: $\pm 16 \%$ at $5 \mathrm{fb}^{-1}$

- WW from data: $\pm 17 \%$ at $5 \mathrm{fb}^{-1}$

- Wt from theory: $\pm 22 \%$

- gg $\rightarrow$ WW from theory: $\quad \pm 30 \%$ 


\section{Vector Boson Fusion qq $\mathrm{H}$}

Motivation: Increase discovery potential at low mass

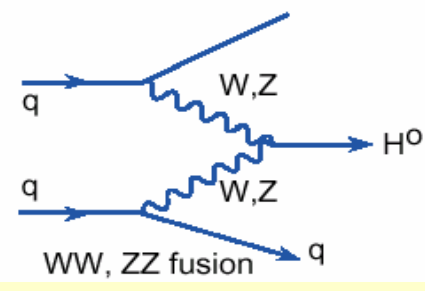
Improve and extend measurement of Higgs boson parameters (couplings to bosons, fermions)

Established (low mass region) by D. Zeppenfeld et al. (1997/98)

Earlier studies: Kleiss \& Stirling (1988);

Dokshitzer, Khoze, Troyan, Sov.J. Nucl. Phys. 46 (1987) 712;

Dokshitzer, Khoze, Sjöstrand, Phys.Lett., B274 (1992) 116.

Distinctive Signature of:

- two high $\mathrm{P}_{\mathrm{T}}$ forward tag jets

- little jet activity in the central region $\Rightarrow$ central jet Veto

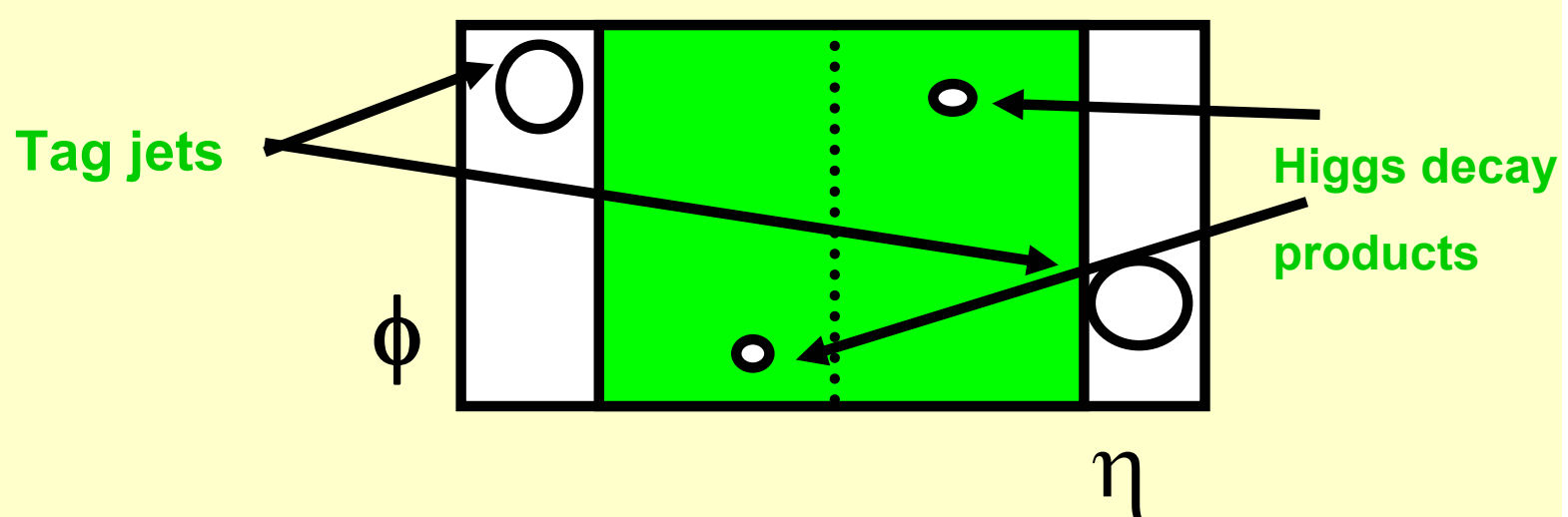

Rapidity distribution of jets in tt and Higgs signal events:

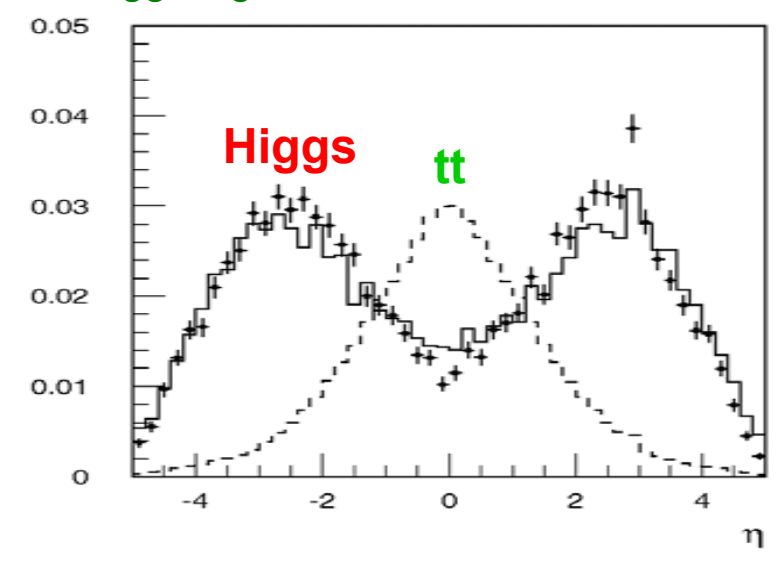




\section{Two search channels at the LHC:}

\section{(i) $q q \mathrm{H} \rightarrow q q \mathrm{~W} \mathrm{~W}^{*}$ $\rightarrow$ qq $e_{v} e_{v}$}

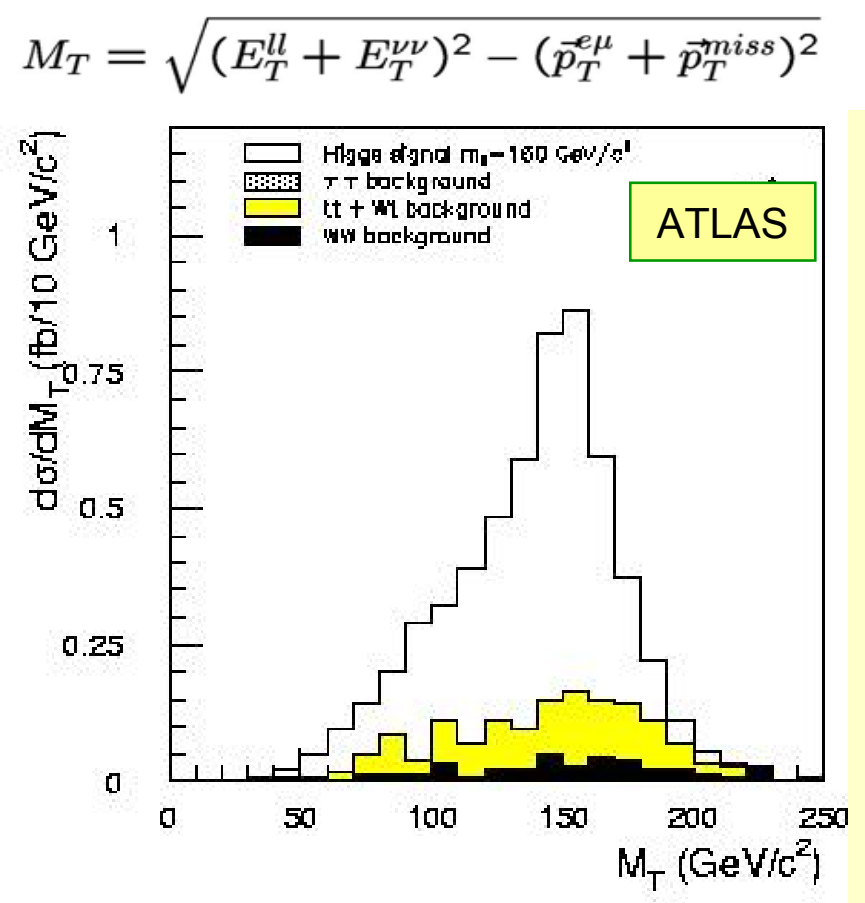

Selection criteria:

- Lepton $\mathrm{P}_{\mathrm{T}}$ cuts and tag jet requirements $\left(\Delta \eta, \mathrm{P}_{\mathrm{T}}\right)$

- Require large mass of tag jet system

- Jet veto (important)

- Lepton angular and mass cuts

Provides $>5 \sigma$ discovery potential for $30 \mathrm{fb}^{-1}$ in mass range

$125<\mathrm{m}_{\mathrm{H}}<200 \mathrm{GeV} / \mathrm{c}^{2} \quad$ (ATLAS)

$140<\mathrm{m}_{\mathrm{H}}<190 \mathrm{GeV} / \mathrm{c}^{2} \quad$ (CMS, new study)

(differences need to be understood)

How reliable is this signal? 


\section{background shape (the experimental approach)}

- Cuts can be relaxed, to get background shape from the data + Monte Carlo:
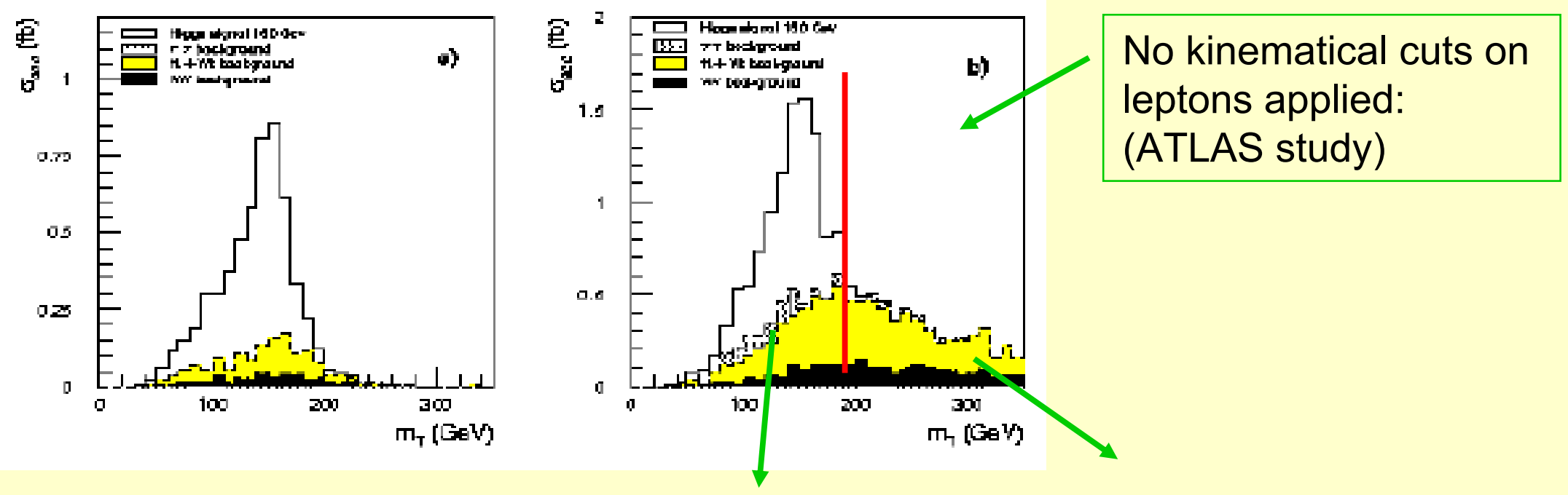

Evidence for spin-0 of the Higgs boson, $\Delta \phi$ distribution

Spin-0 $\rightarrow$ WW $\rightarrow$ $\ell$ v v expect leptons to be close by in space
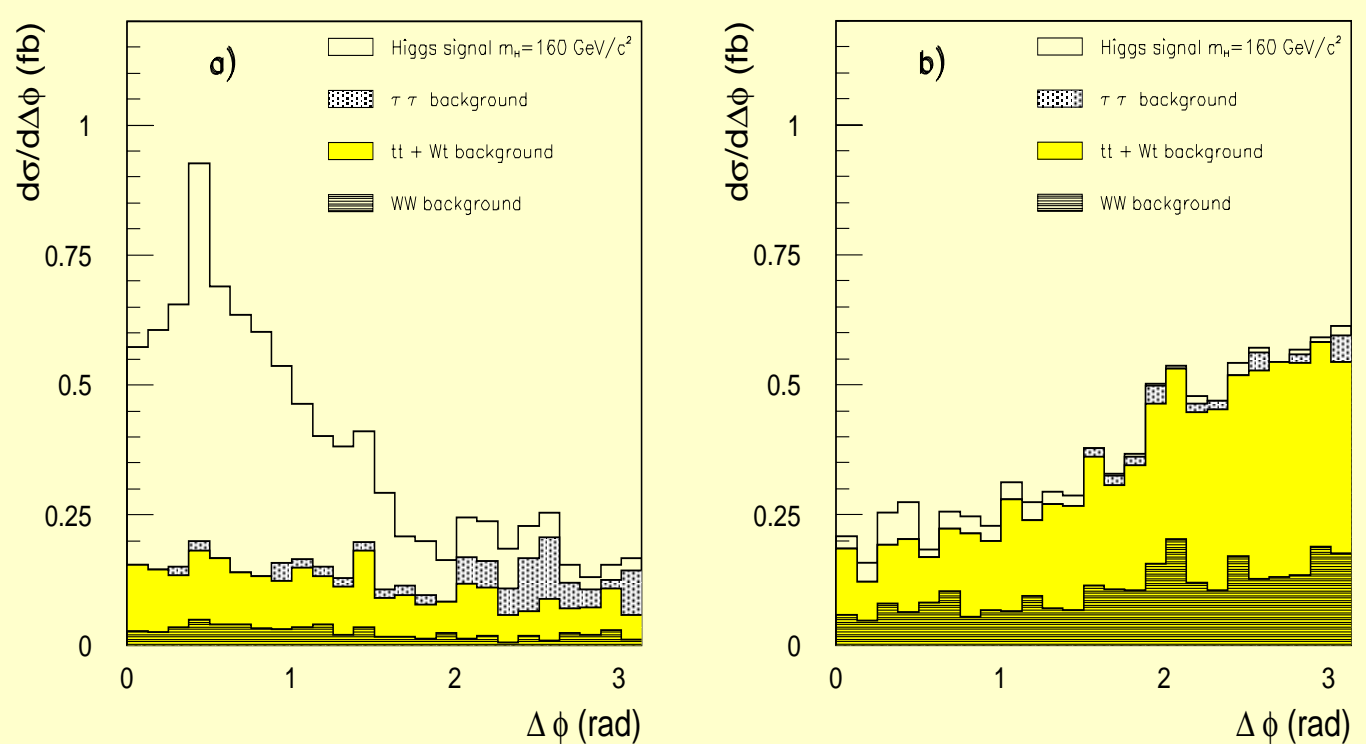


\section{(ii) Results from the first full simulation analysis of qqH $\rightarrow$ qq $\tau \tau \rightarrow$ qq $\ell_{v v}$ had $v$}

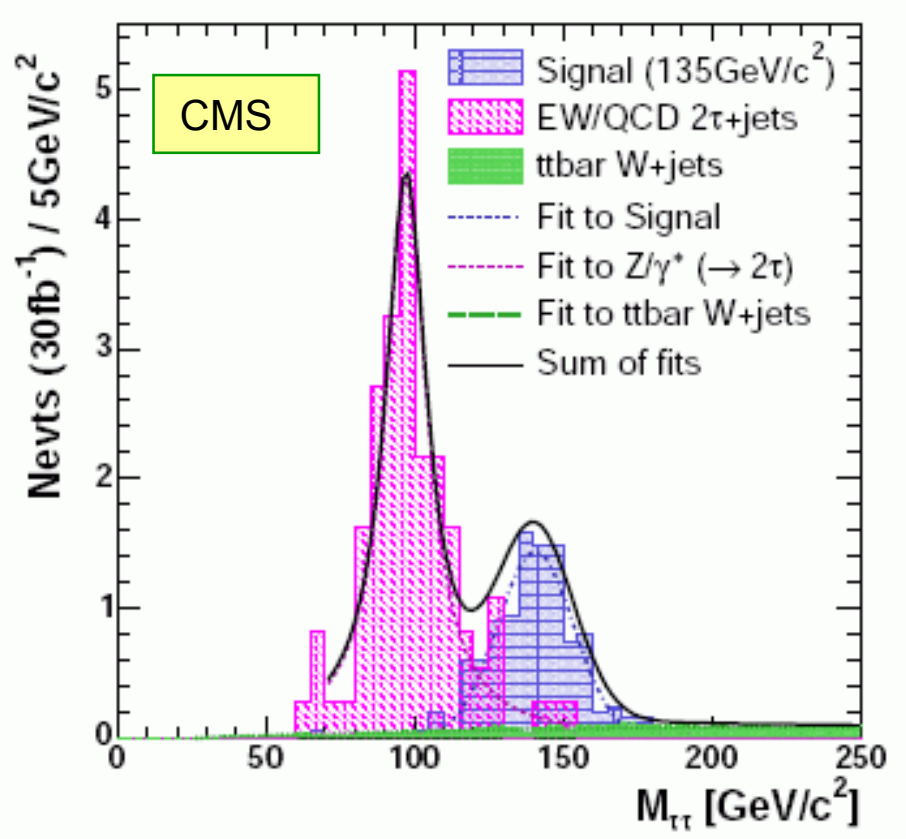

Experimental challenge:

- Identification of hadronic taus

- good $\mathrm{P}_{\mathrm{T}}{ }^{\text {miss }}$ resolution

( $\tau \tau$ mass reconstruction in collinear approximation)

- control of the $Z \rightarrow \tau \tau$ background shape in the high mass region

$\rightarrow$ use data to constrain the background
Signal significance, SM Higgs, e $\mu$ channel:
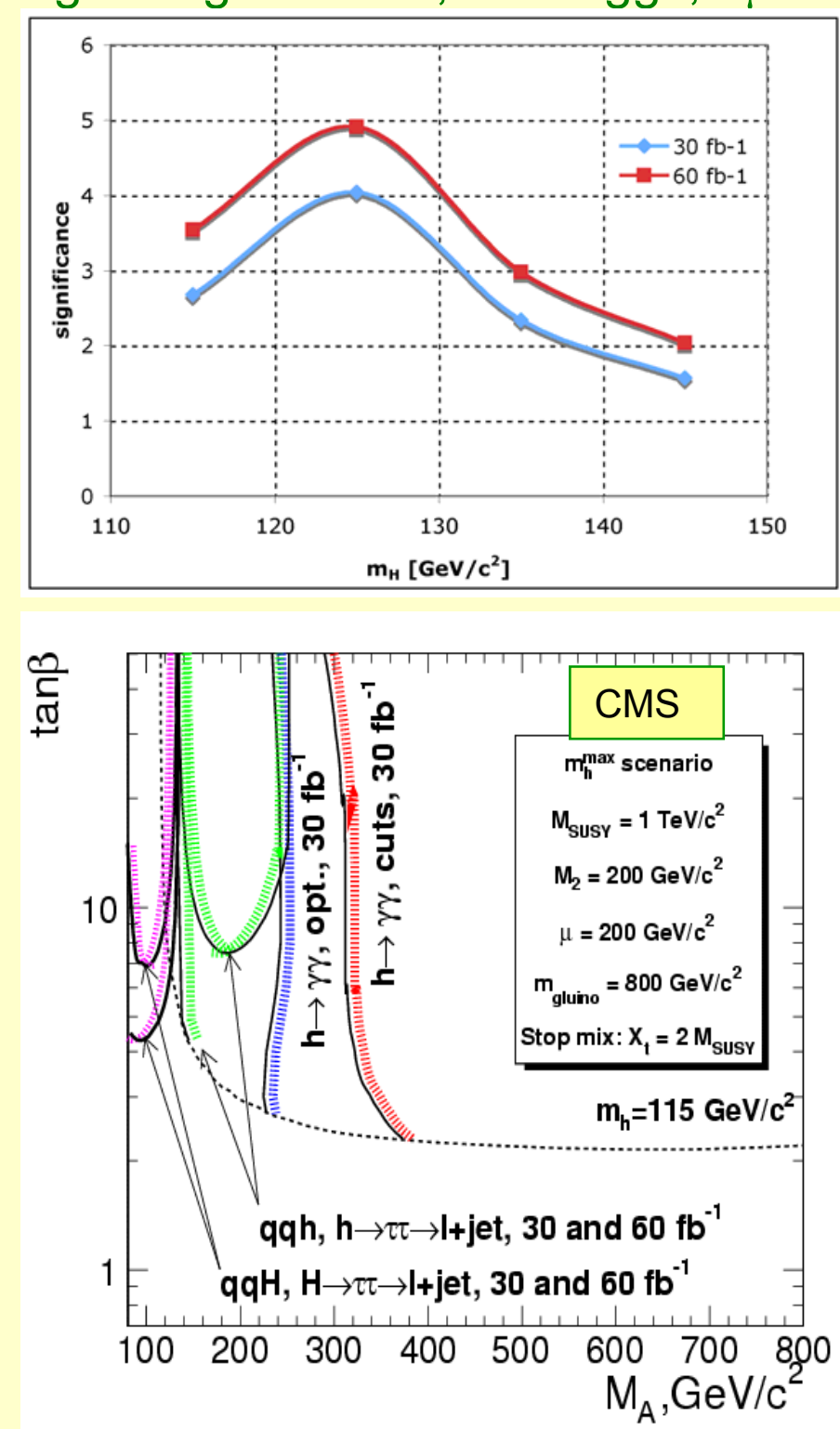

discovery region in the MSSM 


\section{LHC discovery potential for $30 \mathrm{fb}^{-1}$}
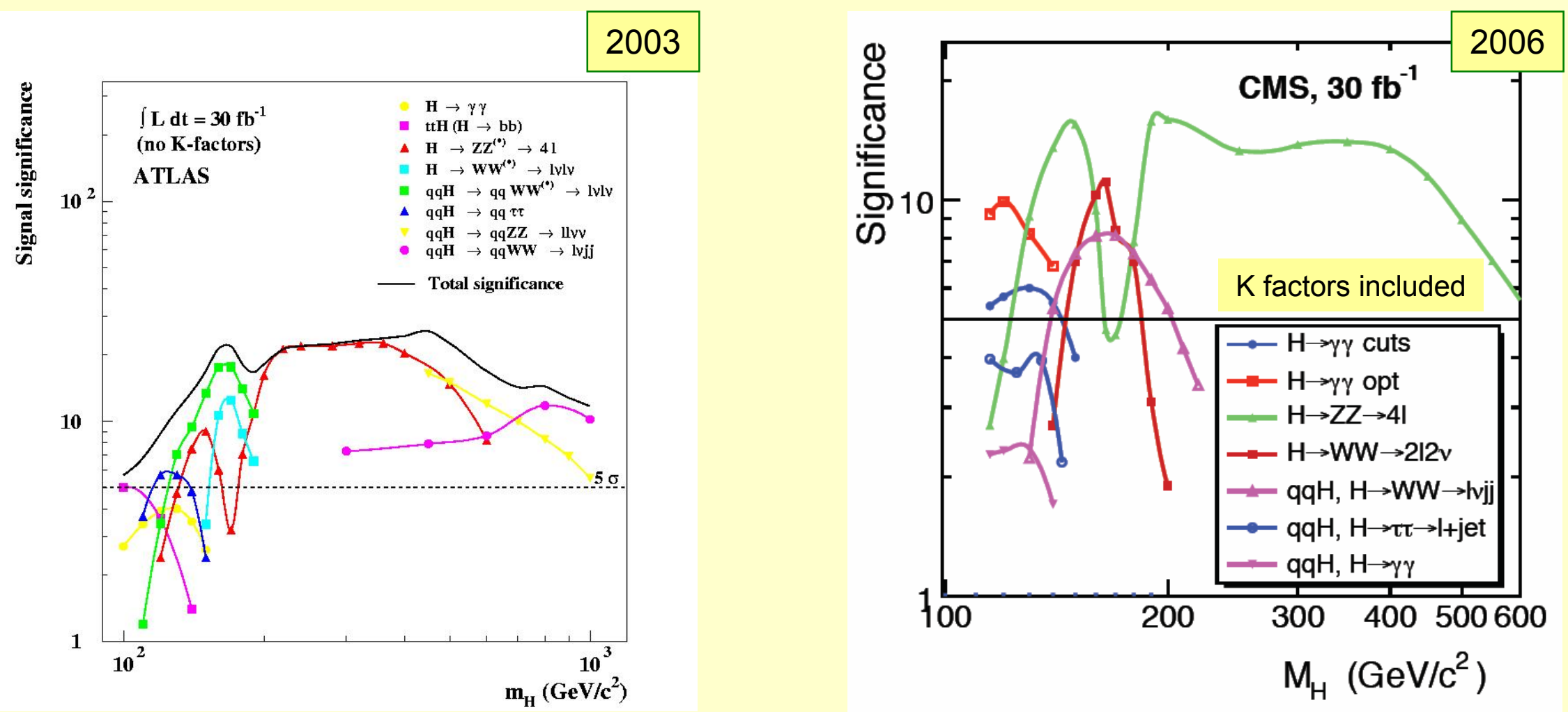

- Full mass range can already be covered after a few years at low luminosity

- Several channels available over a large range of masses Vector boson fusion channels play an important role at low mass !

Important changes w.r.t. previous studies:

- $\mathrm{H} \rightarrow \gamma \gamma \quad$ sensitivity of ATLAS and CMS comparable

- ttH $\rightarrow$ tt bb disappeared in CMS study (updated (ME) background estimates, under study in ATLAS) 


\section{Combined ATLAS + CMS discovery potential}

\section{- Luminosity required for a $5 \sigma$ discovery or a $95 \%$ CL exclusion -}

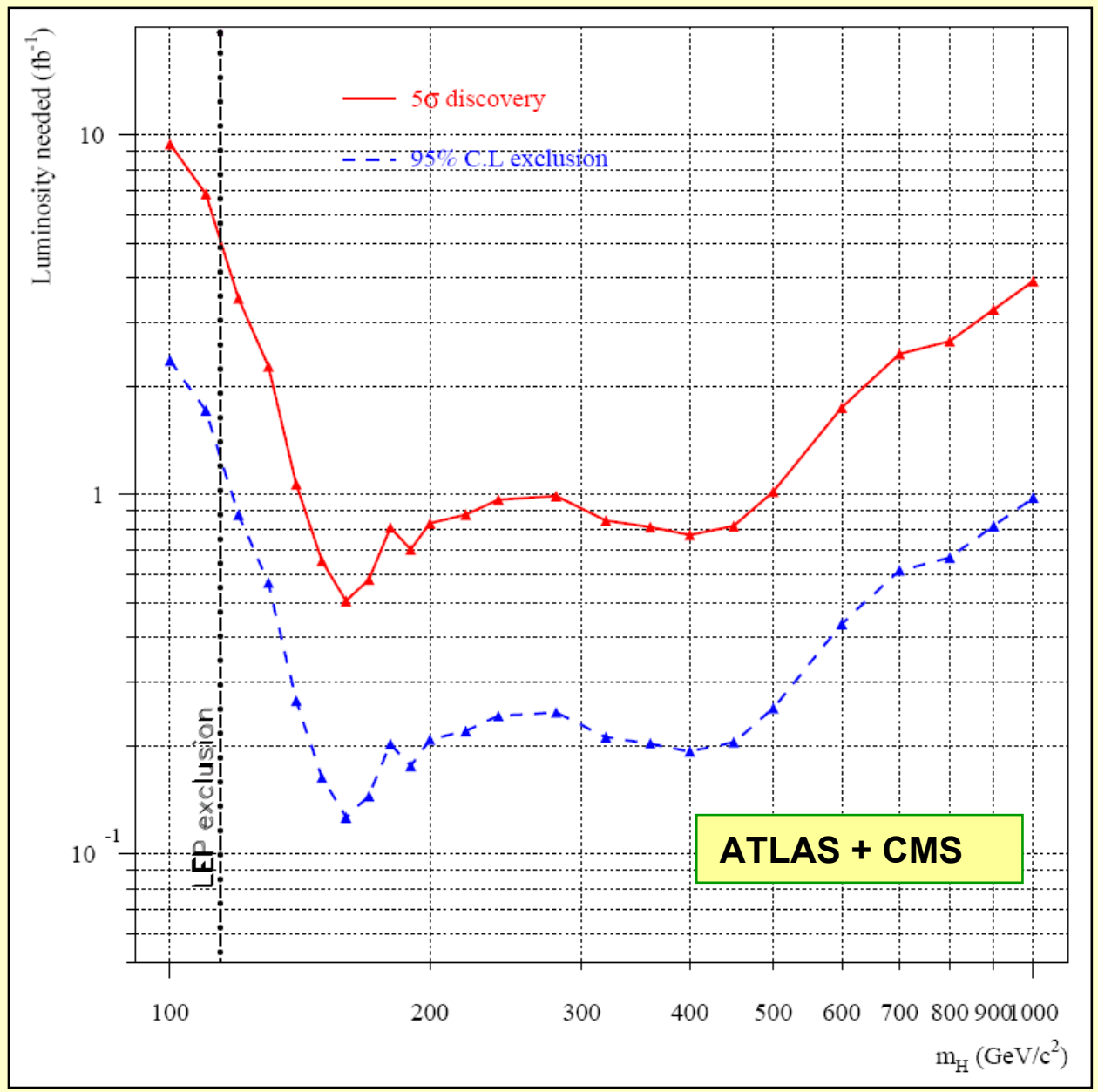

$\sim 5 \mathrm{fb}^{-1}$ needed to achieve a $5 \sigma$ discovery

(well understood and calibrated detector)

$\sim<\mathrm{fb}^{-1}$ needed to set a

95\% CL limit

(low mass $\sim 115 \mathrm{GeV} / \mathrm{c}^{2}$ more difficult)

\section{comments:}

- present curves assume the old $\mathrm{ttH}, \mathrm{H} \rightarrow \mathrm{bb}$ performance

- systematic uncertainties assumed to be luminosity dependent

(no simple scaling, $\sigma \sim \sqrt{ } \mathrm{L}$, possible)

J.J. Blaising et al, Eur. Strategy workshop 


\section{$\overline{\mathrm{tt}} \mathrm{H} \rightarrow \mathrm{t} \overline{\mathrm{t}} \mathrm{b} \overline{\mathrm{b}}$}

Complex final states: $\mathrm{H} \rightarrow \mathrm{bb}, \mathrm{t} \rightarrow \mathrm{bjj}, \mathrm{t} \rightarrow \mathrm{b} \ell \mathrm{v}$

$$
\mathrm{t} \rightarrow \mathrm{b} \ell v, \mathrm{t} \rightarrow \mathrm{b} \ell v
$$

Main backgrounds:

$$
\mathrm{t} \rightarrow \mathrm{bjj}, \mathrm{t} \rightarrow \mathrm{bjj}
$$

- combinatorial background from signal ( $4 \mathrm{~b}$ in final state)

- ttjj, ttbb, ttZ,...

- Wjjjjjj, WWbbjj, etc. (excellent b-tag performance required)

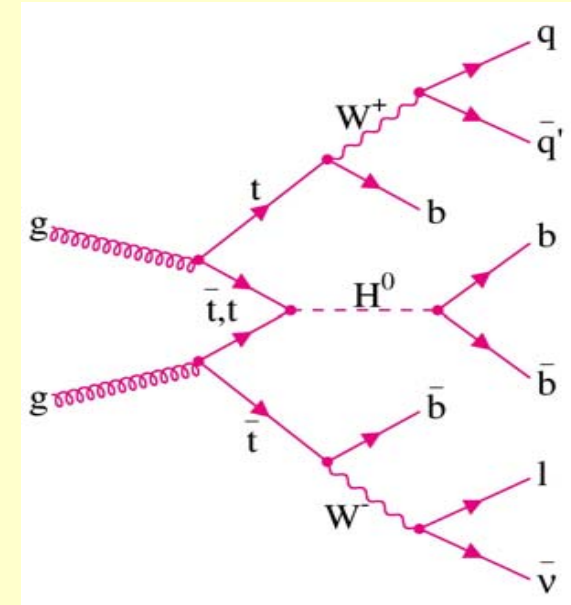

- Updated CMS study (2006): ALPGEN matrix element calculations for backgrounds $\rightarrow$ larger backgrounds (ttij dominant), experimental + theoretical uncertainties, e.g. ttbb, exp. norm. difficult....

$\mathrm{M}(\mathrm{bb})$ after final cuts, $60 \mathrm{fb}^{-1}$

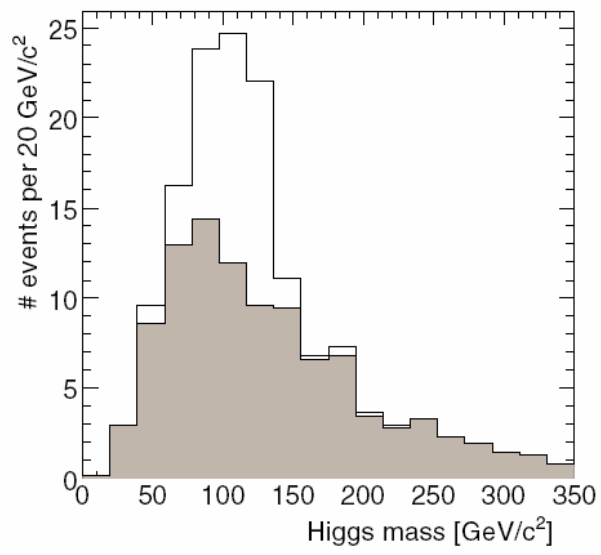

Signal events only

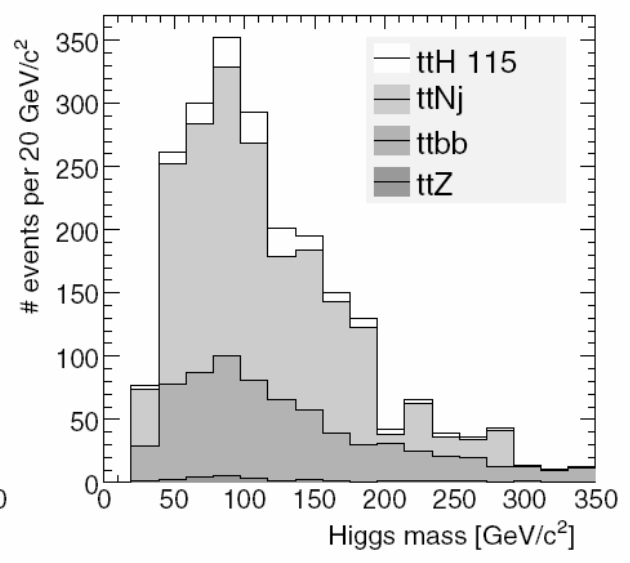

.... backgrounds added

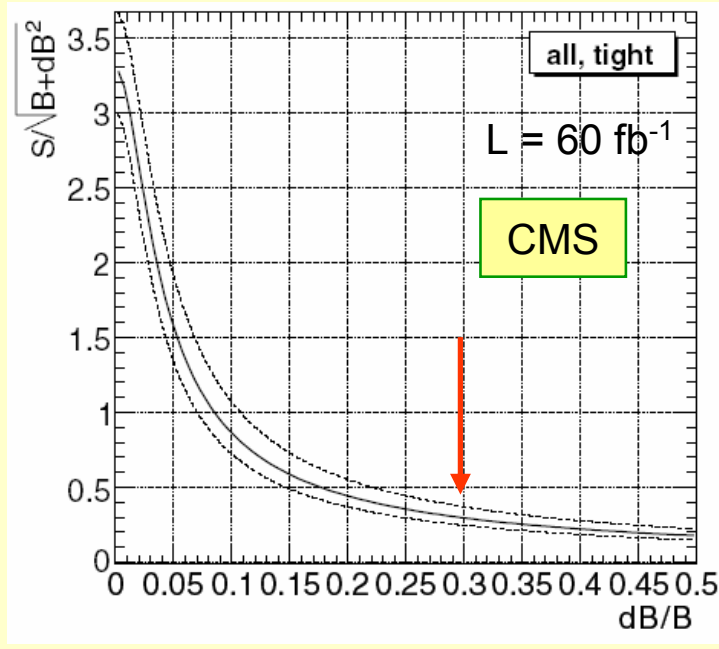

Signal significance as function of background uncertainty

ATLAS study ongoing, results expected by end of the year 


\section{Is it a Higgs Boson?}

-can the LHC measure its parameters?-

\section{Mass}

Higgs boson mass can be measured with a precision of $0.1 \%$

over a large mass range (130 - 450 GeV/c²)

$(\gamma \gamma$ and $Z Z \rightarrow 4 \ell$ resonances, el.magn. calo. scale uncertainty assumed to be $\pm 0.1 \%)$

\section{Couplings to bosons and fermions}

\section{Spin and CP}

Angular distributions in the decay channel $\mathrm{H} \rightarrow \mathrm{ZZ}\left(^{*}\right) \rightarrow 4 \ell$ are sensitive to spin and CP eigenvalue

C.P. Buszello et al. Eur. Phys. J. C32 (2003) 209;

S. Y. Choi et al., Phys. Lett. B553 (2003) 61.

$\rightarrow$ ATLAS and CMS studies on $\mathrm{H} \rightarrow \mathrm{ZZ} \rightarrow 4 \ell$

+ new studies using VBF (CP from tagging jets) in ATLAS $\quad(\rightarrow$ Talks in parallel sessions)

\section{Higgs self coupling}

Possible channel: $\mathrm{gg} \rightarrow \mathrm{HH} \rightarrow \mathrm{WW}$ WW $\rightarrow$ ev jj $\ell v \mathrm{jj} \quad$ (like sign leptons)

Small signal cross sections, large backgrounds from $t t, W W, W Z, W W W, t t t, ~ W t t, \ldots$

$\Rightarrow$ no significant measurement possible at the LHC very difficult at a possible SLHC $\left(6000 \mathrm{fb}^{-1}\right)$ limited to mass region around $160 \mathrm{GeV} / \mathrm{c}^{2}$ (update will appear soon) 


\section{Measurement of Higgs Boson Couplings}

Global likelihood-fit (at each possible Higgs boson mass) Input: measured rates, separated for the various production modes

Output: Higgs boson couplings, normalized to the WW-coupling
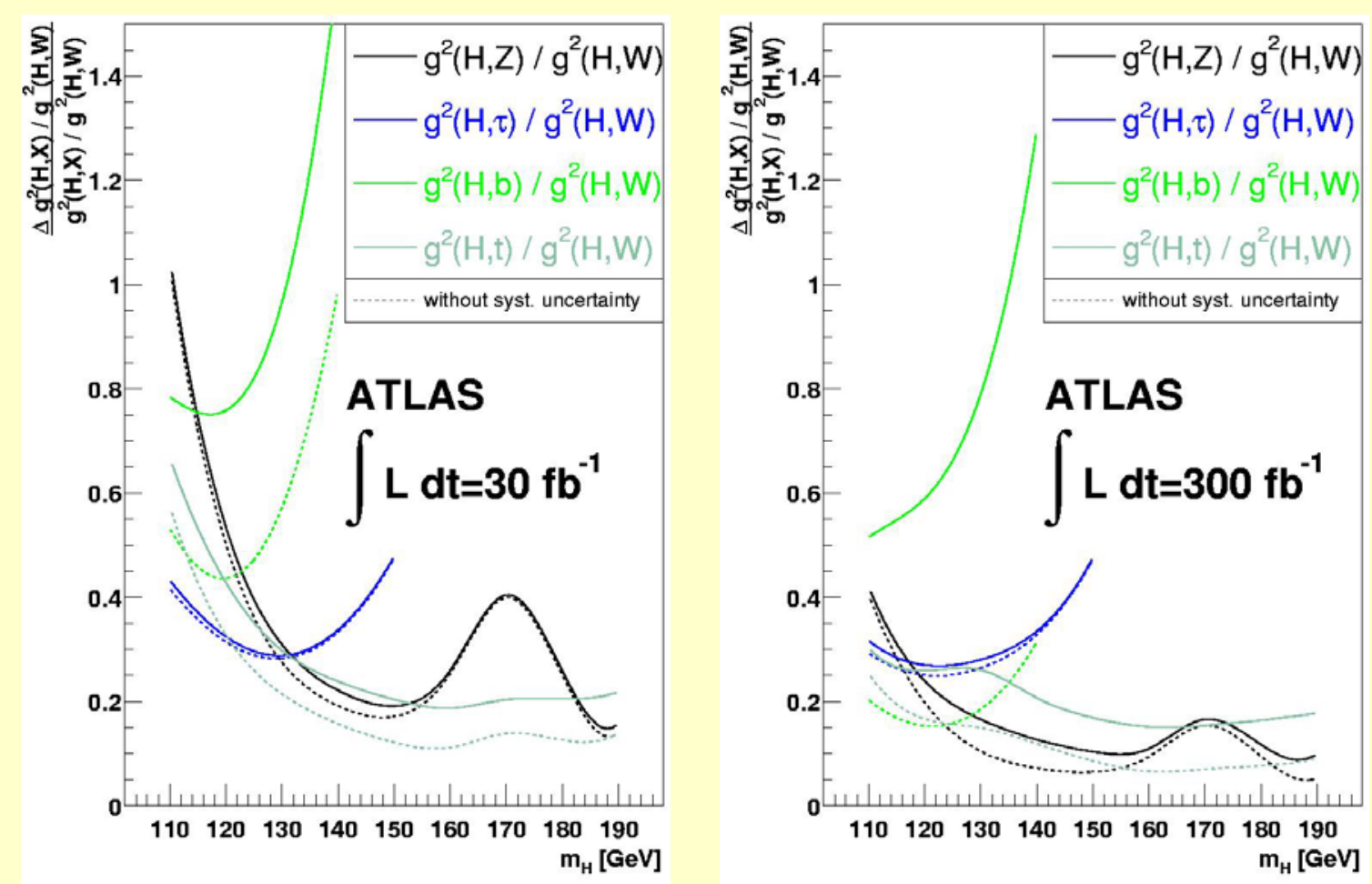

Relative couplings can be measured with a precision of $~ 20 \%$ (for $300 \mathrm{fb}^{-1}$ ) 


\section{The Higgs Sector}

in the MSSM

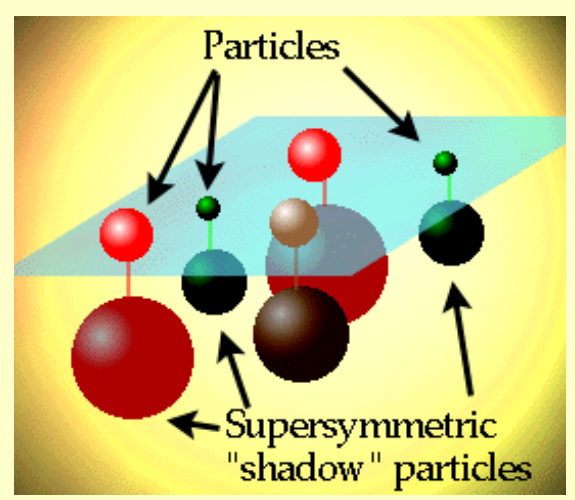




\section{MSSM Higgs bosons $h, H, A, H^{ \pm}$}

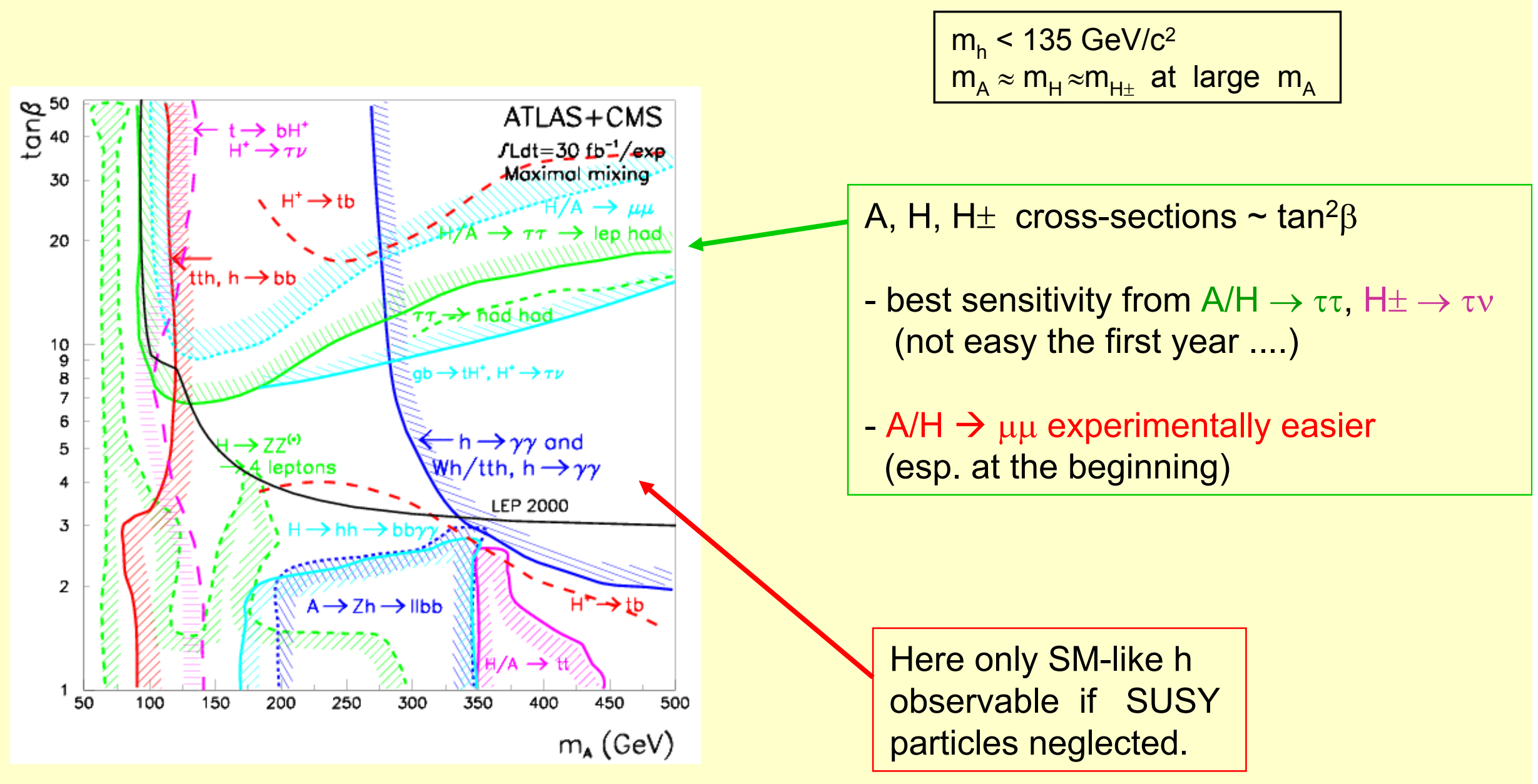

* Validated by CMS TDR full simulation studies * 


\section{Some examples of updated MSSM studies}

\section{CMS: $\quad \mathrm{A} / \mathrm{H} \rightarrow \mu \mu$}
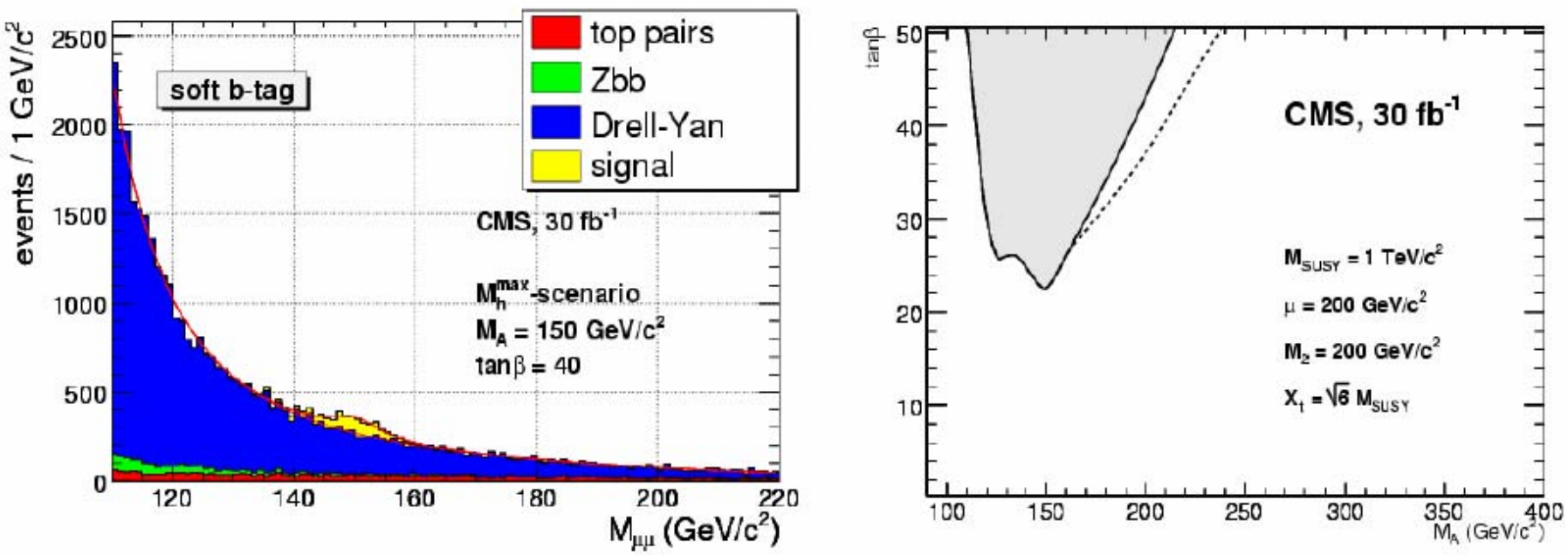

ATLAS: Charged Higgs boson searches $\mathrm{H}^{+} \rightarrow \tau \nu$ decay mode

Production modes:

$\mathrm{t} \rightarrow \mathrm{H}^{+} \mathrm{b} \quad \mathrm{gg} \rightarrow \mathrm{tbH}^{+} \quad \mathrm{gb} \rightarrow \mathrm{tH}^{+}$

Consistent treatment of the transition region $m_{H^{+}} \sim m_{t}$

Event generator MATCHIG

$(\rightarrow$ talk in parallel session)

$5 \sigma$ discovery contours for 30 and $100 \mathrm{fb}^{-1}$

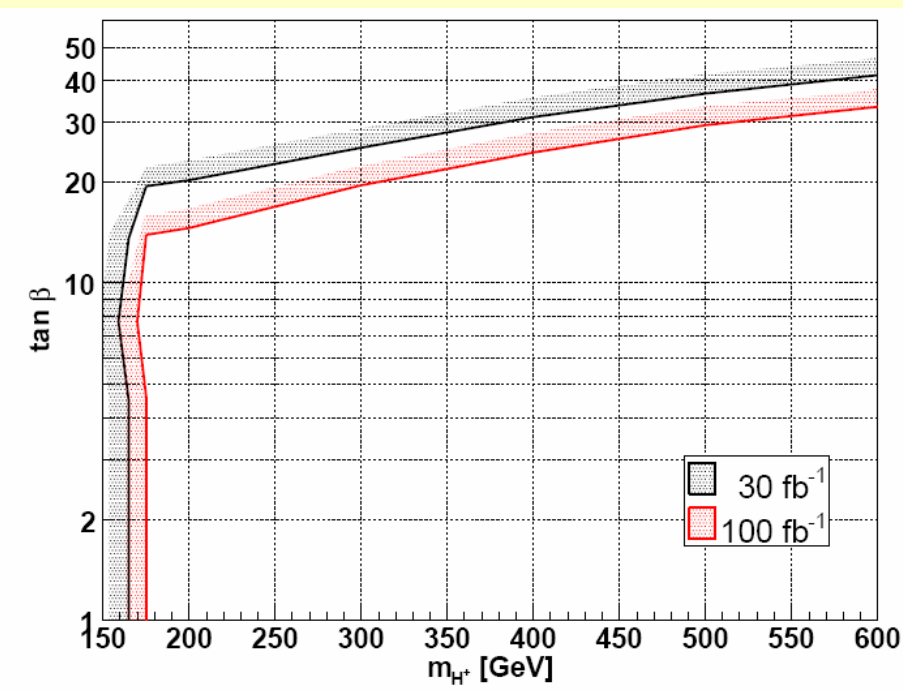




\section{Updated MSSM scan for different benchmark scenarios}

Benchmark scenarios as defined by M.Carena et al. (h mainly affected)

ATLAS preliminary, $30 \mathrm{fb}^{-1}, 5 \sigma$ discovery
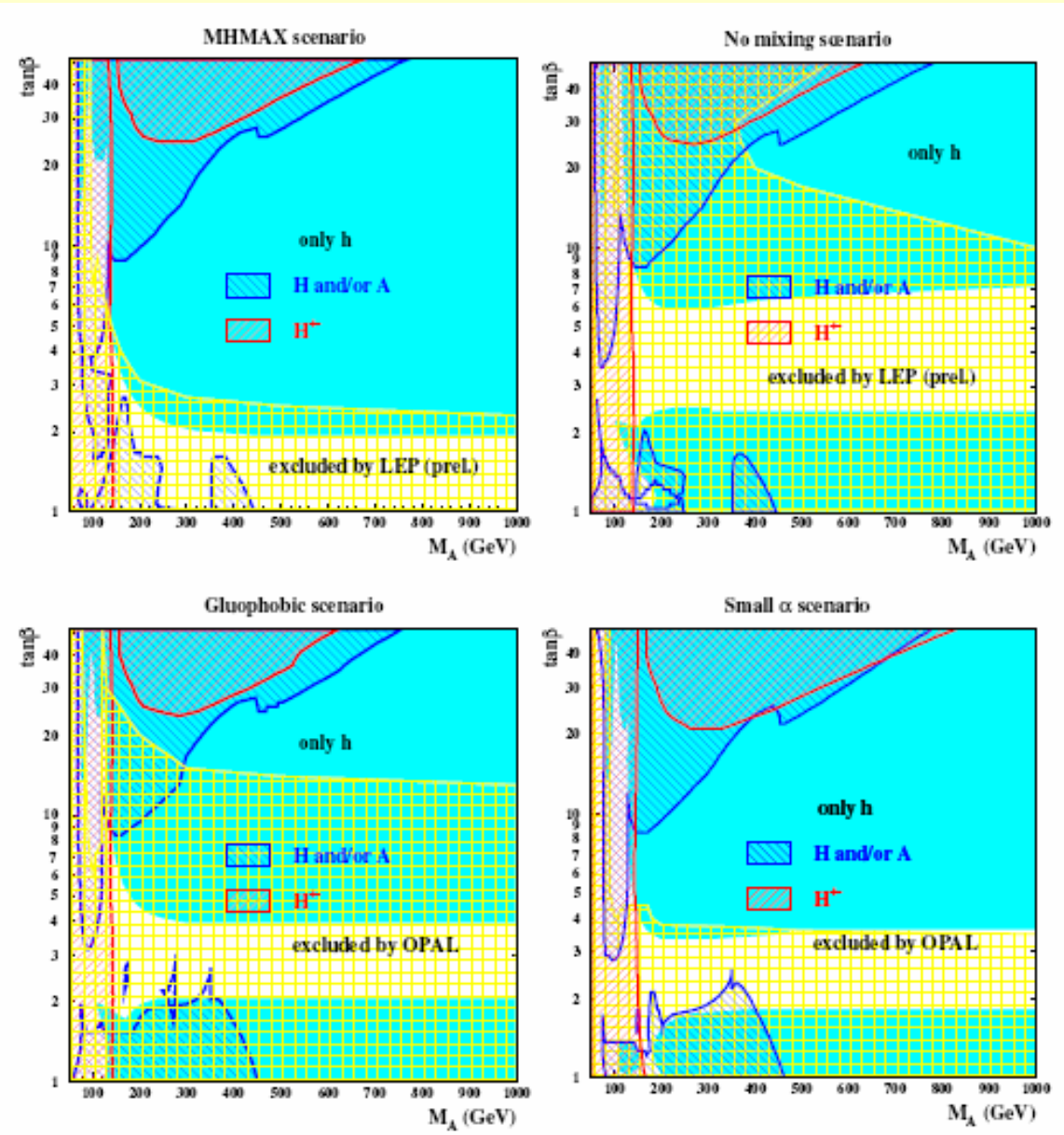

MHMAX scenario $\quad\left(\mathrm{M}_{\mathrm{SUSY}}=1 \mathrm{TeV} / \mathrm{c}^{2}\right)$ maximal theoretically allowed region for $m_{h}$

Nomixing scenario $\quad\left(\mathrm{M}_{\mathrm{SUSY}}=2 \mathrm{TeV} / \mathrm{c}^{2}\right)$ (1TeV almost excl. by LEP ) small $m_{h} \rightarrow$ difficult for LHC

Gluophobic scenario $\left(\mathrm{M}_{\mathrm{SUSY}}=350 \mathrm{GeV} / \mathrm{c}^{2}\right)$ coupling to gluons suppressed (cancellation of top + stop loops) small rate for $\mathrm{g} g \rightarrow \mathrm{H}, \mathrm{H} \rightarrow \gamma \gamma$ and $\mathrm{Z} \rightarrow 4 \ell$

Small $\alpha$ scenario $\quad\left(\mathrm{M}_{\text {SUSY }}=800 \mathrm{GeV} / \mathrm{c}^{2}\right)$ coupling to $b$ (and $\mathbf{t}$ ) suppressed (cancellation of sbottom, gluino loops) for large $\tan \beta$ and $\mathrm{M}_{\mathrm{A}} 100$ to $500 \mathrm{GeV} / \mathrm{c}^{2}$ 


\section{Higgs search at the LHC in CP-violating scenarios}

- CP conservation at Born level, but CP violation via complex $\mathbf{A}_{\mathbf{t}}, \mathbf{A}_{\mathbf{b}}, \mathbf{M} \ldots$.

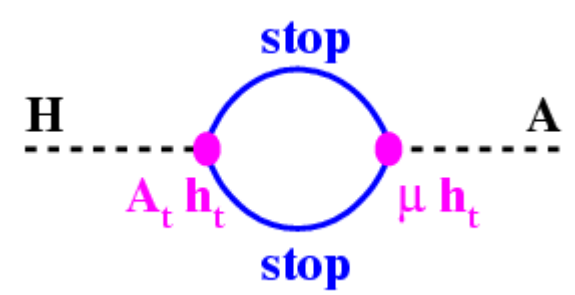

- CP eigenstates $h, A, H$ mix to mass eigenstates $H_{1}, H_{2}, H_{3}$

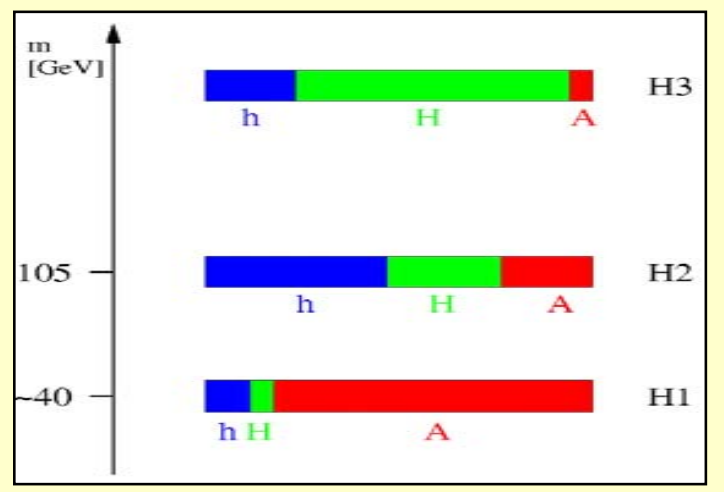

- Effect maximized in a defined benchmark scenario (CPX)

(M. Carena et al., Phys.Lett. B 495155 (2000))

$\arg \left(A_{t}\right)=\arg \left(A_{b}\right)=\arg \left(M_{\text {gluino }}\right)=90^{\circ}$

- No lower mass limit for $\mathrm{H}_{1}$ from LEP !

(decoupling from the $Z$ )

details depend on $\mathrm{m}_{\text {top }}$ and on theory model

(FeynHiggs vs. CPsuperH)

$\mathrm{m}_{\text {top }}=169.3 \mathrm{GeV} / \mathrm{c}^{2}$

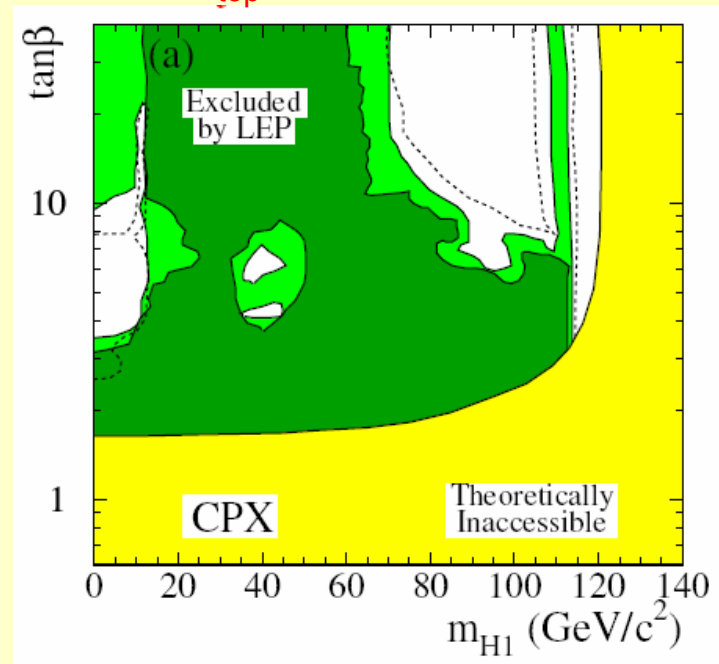

$\mathrm{m}_{\text {top }}=174.3 \mathrm{GeV} / \mathrm{c}^{2}$

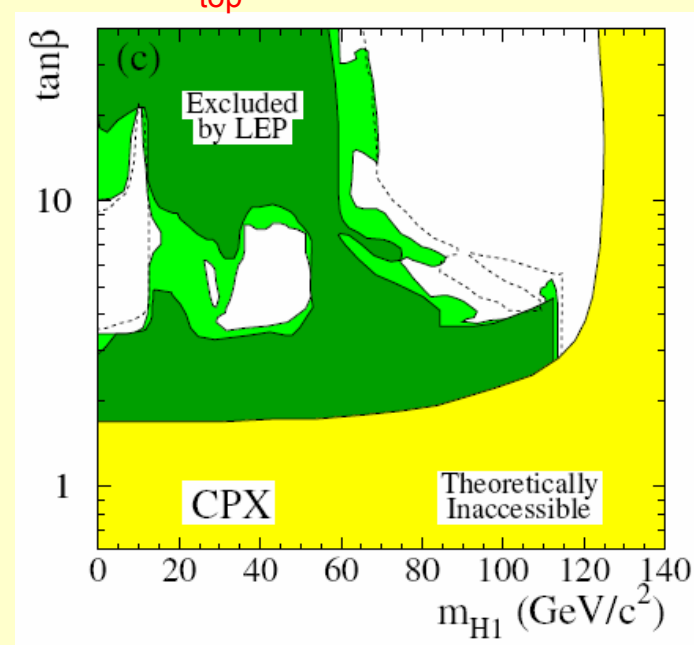




\section{MSSM discovery potential for the CPX scenario}
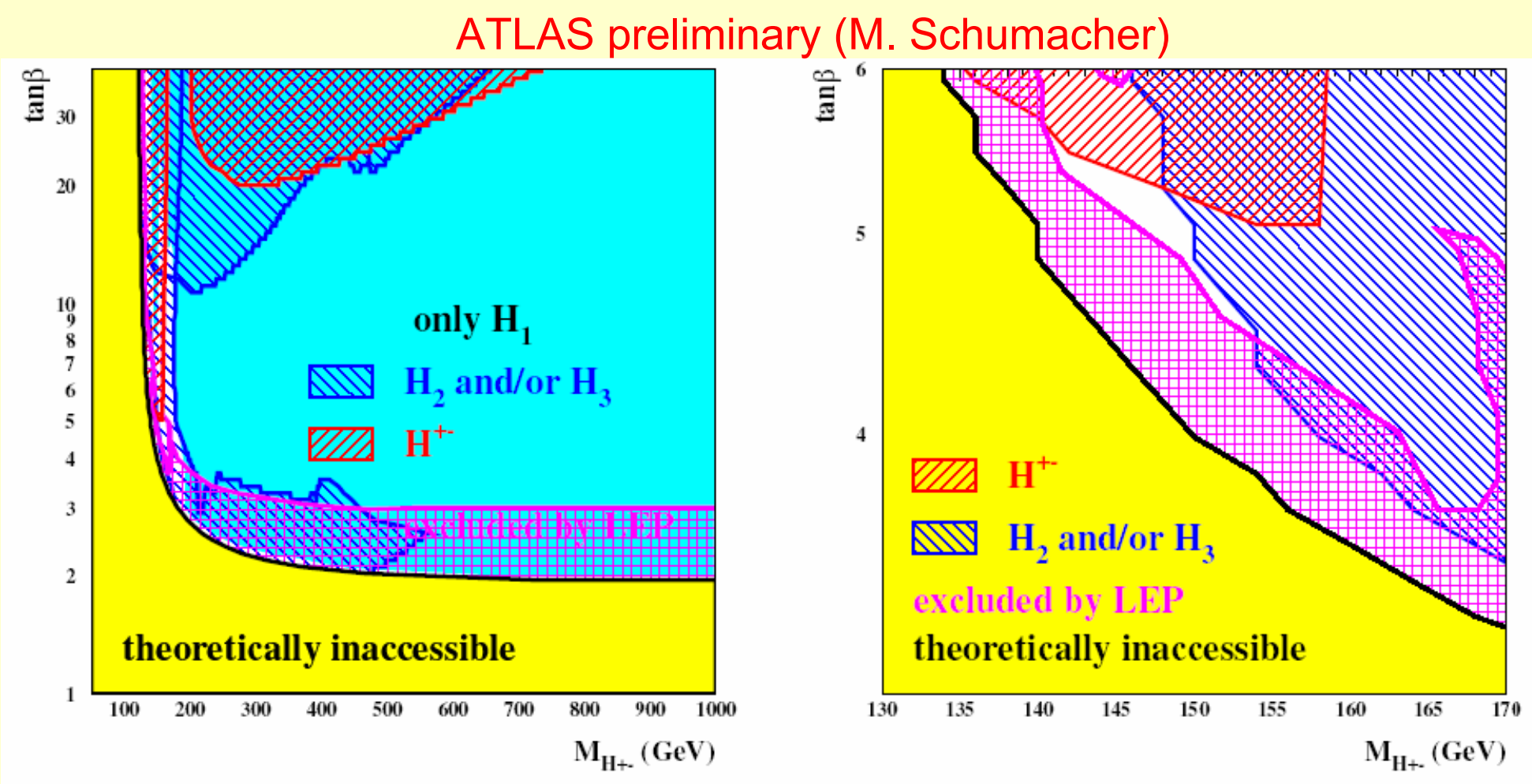

- Large fraction of the parameter range can be covered, however, small hole at (intermediate $\tan \beta$, low $\mathrm{m}_{\mathrm{H}_{+}}$) corresponding to low $\mathrm{m}_{\mathrm{H}_{1}}$

- More studies needed, e.g. investigate lower $\mathrm{H}_{1}$ masses, additional decay channels:

$\mathrm{tt} \rightarrow \mathrm{Wb} \mathrm{H}+\mathrm{b} \rightarrow \ell v b \mathrm{WH}_{1} \mathrm{~b}, \mathrm{H}_{1} \rightarrow \mathrm{bb}$ 


\section{Invisible Higgs decays?}

Possible searches: $\quad$ tt $\mathrm{H} \rightarrow \ell v b q q b+\mathrm{P}_{T}$ miss

$$
\begin{array}{ll}
\mathrm{ZH} \rightarrow \mathrm{ll} & +\mathrm{P}_{\mathrm{T}}^{\text {miss }} \\
\mathrm{qqH} \rightarrow \mathrm{qq} & +\mathrm{P}_{\mathrm{T}}^{\text {miss }}
\end{array}
$$

- J.F. Gunion, Phys. Rev. Lett. 72 (1994)

- D. Choudhury and D.P. Roy, Phys. Lett. B322 (1994)

- O. Eboli and D. Zeppenfeld, Phys. Lett. B495 (2000)

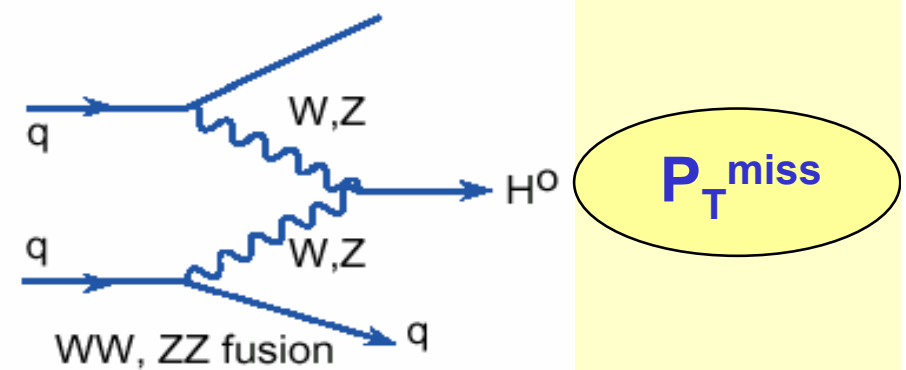

All three channels have been studied:

key signature: excess of events above SM backgrounds with large $P_{T}{ }^{\text {miss }}$ ( $>100 \mathrm{GeV} / \mathrm{c}$ )

$$
\text { Sensitivity: } \quad \xi^{2}=\operatorname{Br}(H \rightarrow \operatorname{Inv} .) \frac{\sigma_{q q \rightarrow q q H}}{\sigma_{q q \rightarrow q q H} \mid S M}
$$

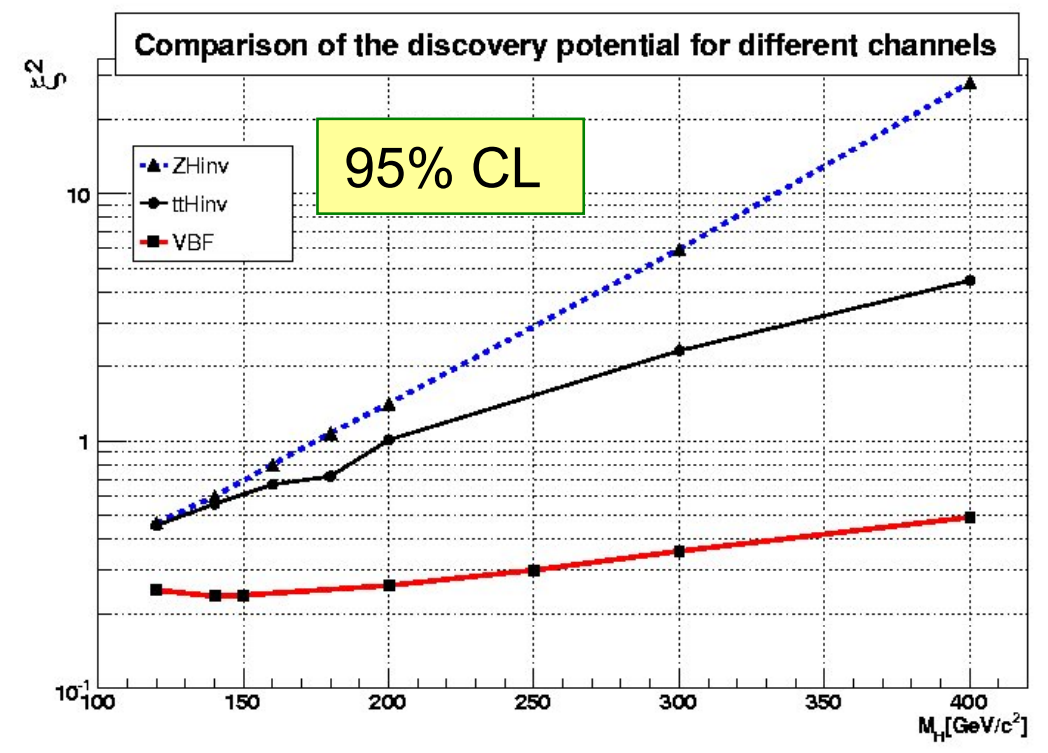

ATLAS preliminary

\section{Problems / ongoing work:}

- $\mathrm{ttH}$ and $\mathrm{ZH}$ channels have low rates

- More difficult trigger situation for $q q \mathrm{H}$

- backgrounds need to be precisely known (partially normalization using ref. channels possible)

- non SM scenarios are being studied at present first example: SUSY scenario 


\section{Conclusions}

- The LHC experiments are well set up to explore the existence of a Standard Model or MSSM Higgs bosons and are well prepared for unexpected scenarios

- The full Standard Model mass range and the full MSSM parameter space can be covered (CP-conserving models)

in addition: important parameter measurements (mass, spin, ratio of couplings) can be performed, vector boson fusion channels are important

more difficult: - invisible Higgs boson decays or NMSSM models

- measurement of Higgs boson self coupling

- LHC data will hopefully soon give guidance to theory and to future experiments

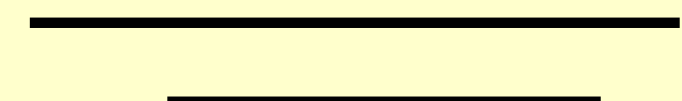

